Article

\title{
A Pontryagin Minimum Principle-Based Adaptive Equivalent Consumption Minimum Strategy for a Plug-in Hybrid Electric Bus on a Fixed Route
}

\author{
Shaobo Xie ${ }^{1,2, *}$, Huiling Li ${ }^{1}$, Zongke Xin ${ }^{1}$, Tong Liu ${ }^{1}$ and Lang Wei ${ }^{1}$ \\ 1 School of Automotive Engineering, Chang'an University, Southern 2nd Road, Xi'an 710064, China; \\ 2015122030@chd.edu.cn (H.L.); 2016222035@chd.edu.cn (Z.X.); 2014222050@chd.edu.cn (T.L.); \\ qch_1@chd.edu.cn (L.W.) \\ 2 National Engineering Laboratory for Electric Vehicles, Beijing Institute of Technology, \\ No. 5 South Zhongguancun Street, Haidian District, Beijing 100081, China \\ * Correspondence: xieshaobo@chd.edu.cn; Tel.: +86-029-8233-4987
}

Received: 1 August 2017; Accepted: 8 September 2017; Published: 11 September 2017

\begin{abstract}
When developing a real-time energy management strategy for a plug-in hybrid electric vehicle, it is still a challenge for the Equivalent Consumption Minimum Strategy to achieve near-optimal energy consumption, because the optimal equivalence factor is not readily available without the trip information. With the help of realistic speeding profiles sampled from a plug-in hybrid electric bus running on a fixed commuting line, this paper proposes a convenient and effective approach of determining the equivalence factor for an adaptive Equivalent Consumption Minimum Strategy. Firstly, with the adaptive law based on the feedback of battery SOC, the equivalence factor is described as a combination of the major component and tuning component. In particular, the major part defined as a constant is applied to the inherent consistency of regular speeding profiles, while the second part including a proportional and integral term can slightly tune the equivalence factor to satisfy the disparity of daily running cycles. Moreover, Pontryagin's Minimum Principle is employed and solved by using the shooting method to capture the co-state dynamics, in which the Secant method is introduced to adjust the initial co-state value. And then the initial co-state value in last shooting is taken as the optimal stable constant of equivalence factor. Finally, altogether ten successive driving profiles are selected with different initial SOC levels to evaluate the proposed method, and the results demonstrate the excellent fuel economy compared with the dynamic programming and PMP method.
\end{abstract}

Keywords: plug-in hybrid electric bus; adaptive equivalent consumption minimum strategy; Pontryagin's Minimum Principle; shooting method; realistic speeding profiles

\section{Introduction}

\subsection{Ground and Literature Review}

The development and large-scale application of electrified vehicles in recent years have been a surging trend worldwide to better cope with the increasing air pollution and energy shortage as well as to achieve the technological upgrading of automotive sector. Particularly, the plug-in hybrid electric bus, which can improve the fuel economy, reduce the exhaust emissions, and also overcome the range anxiety during long-distance trips, has been extensively applied in the public transport field and exhibited a promising future [1,2].

As a multiple sources system for the plug-in hybrid electric vehicle (PHEV), a variety of energy management strategies (EMSs) have been developed including the rule-based method, or the charging-depleting and charging-sustaining (CD-CS) strategy [3], the global and instantaneous 
optimization methods [4,5], and the artificial intelligence algorithm [6]. In general, the most common on-broad strategy is on the basis of either the threshold rules or the rules governed by the fuzzy logic or even extracted from global optimization results $[7,8]$.

Known as a typical global algorithm, dynamic programming (DP) has been widely utilized to optimize the EMS, making it a benchmark to evaluate other methods $[9,10]$. However, the disadvantage to this algorithm is the heavy computational burden, because of the massive discrete state and input points together with the interpolating calculation during the iterative process. Another global approach is based on Pontryagin's Minimum Principle (PMP), in which the optimal power distribution policy is searched by minimizing the Hamilton function while respecting the SOC upper and lower bounds as well as other physical constraints [11]. In contrast to the DP method, PMP algorithm has greatly improved the computational efficiency [12]. Though the PMP-based energy management issue can be converted into a two-point boundary value problem (TPBVP) [13], it is still not easy to obtain the numerical solution directly, because of the complex co-state dynamics involving the partial differential equation associated with the nonlinearity of the battery model [10]. Many approaches have been proposed to deal with this problem, for instance, [14] regarded the co-state variable as a constant with the assumption that the battery internal resistance and open-circuit voltage keep almost unchangeable for HEVs. Xu overcome this obstacle by fitting the battery open-circuit voltage and internal resistance as a polynomial of SOC [15]. Li proposed to adopt the pre-defined lookup tables in Matlab/Simulink to cope with this issue [16]. Nevertheless, it is necessary and critical to obtain the numerical solution in a direct manner so as to acquire the initial co-state value and optimal co-state trajectory. Furthermore, both the DP and PMP algorithms cannot be carried out without a-priori knowledge of the driving cycle; that is, the complete trip information must be given in advance, hence leading to an impractical EMS.

Unlike the DP and PMP algorithms, the Equivalent Consumption Minimum Strategy (ECMS) as an instantaneous method was originally proposed to develop the EMS for HEVs, given that the consumed electrical energy will be supplemented by the on-board generator [17], and this strategy is further extended to PHEVs, in which the stored electricity will be used up finally during a blended discharging to achieve the minimum energy consumption. Therefore, the ECMS for HEVs and PHEVs mainly differ in the SOC trajectory. To be more specific, the reference SOC for HEVs always keeps a constant level while the SOC for PHEVs is expected to decline along the travel distance during a blended charging, provided that the vehicle will run out of the battery electricity at the end of the trip [18].

For HEVs, the equivalence factor (EF) is often considered as a constant, under the assumption that the battery almost maintains unchanged internal resistance and open circuit voltage with respect to SOC $[10,14]$. The EF can be further modified by a weighting of such two constants for the charging and discharging respectively [19]. To be a more feasible strategy under unknown driving circumstances, several novel adaptive ECMSs have been recently suggested for HEVs. Onori [20] recognized three categories of adaptive laws to update the EF including the driving cycle prediction, driving pattern recognition (DPR) and feedback of battery SOC, and furthermore discussed three adaptive methods based on SOC feedback. Likewise, Gu [21] employed the DPR for a HEV to smartly tune the EF in accordance with the currently recognized running style. Also, Yokoi [22] suggested constructing a driving pattern database with a clustering method for a specific driving course. When developing the ECMS for the PHEVs, the EF has to be tuned according with the driving cycle. To adapt to the trip with unfixed distances, References $[13,23]$ proposed an adaptive PMP supervisory controller with a look-up table, via which the corresponding optimal EF can be selected based on the required information of average speed and estimated trip distance. Moreover, a map-based ECMS is illustrated to deal with different route distances, in which a proportional and integral (PI) controller is implemented to follow the reference SOC and the optimal constant EF is estimated for different route length [24]. In addition, Larsson [25] studied the benefits of the route recognition for a family car, by which the EMS mode can switch from the CD-CS to blended discharging mode by matching the ongoing driving trip against a candidate route. For a parallel PHEV running on a city-bus route, $\mathrm{Li}$ [26] proposed a 
correctional DP-based EMS. Besides, based on the DP solution, Han [27] presented an approach of extracting the globally optimal EF trajectory for an adaptive ECMS strategy, and further investigated the state inequality constrain during the hilly road environment.

Another commonly utilized real-time method for PHEVs is the predictive EMS. With the Markov chain assumption, it is capable of realizing the speed forecasting over the moving horizon in which the DP or PMP algorithm can be executed to acquire a near-optimal solution [28-30]. He [31] performed a stochastic DP for a serial-parallel PHEV by comparing three SOC restrain methods in the receding horizon. Moreover, Li [32] proposed to modify the stochastic model predictive control with the ECMS as well as the driving behavior classification for a commuting bus. In addition, the artificial intelligence (AI) method is also employed to forecast the future speed in the receding horizon, and the significant advantage of the AI technology lies in the powerful leaning ability from the massive training samples. For example, Sun $[33,34]$ performed the DP and ECMS over the predictive horizon by using the back propagation neural network.

\subsection{Motivation}

Despite the availability of various EMSs, including the ECMS for the PHEV, i.e., the adaptive Equivalent Consumption Minimum Strategy (A-ECMS) based on the SOC feedback, it is urgent to find a convenient and robust way of determining all the parameters in EF, especially the stable constant of EF. And the motivation of this paper has following aspects. Firstly, to develop an A-ECMS for a plug-in hybrid electric bus (PHEB) on a fixed route, the available information can be fully utilized such as the route length, which always remains within a limited finite range, and the daily speeding data, which can reflect the running environment characteristics. Secondly, the co-state dynamics in PMP algorithm has been considered as an effective means to produce the optimal EF, however, the detailed procedures to solve the PMP strategy, for instance, by using the shooting method, is seldom presented. Particularly, how to efficiently capture the optimal co-state value is still a challenging issue, because the tuning of the initial co-state value in each shooting process, if without a proper method, will lead to repeated trials, which will inevitably weaken the implement of the A-ECMS. Therefore, it is necessary and important to study how to obtain the numerical solution of PMP strategy, including the choice of initial co-state value and corresponding calibrating method. Thirdly, though most of proposed A-ECMSs exhibit excellent performance, they are almost examined based on a single initial SOC level. In fact, the initial SOC varies during each trip and cannot keep the same level due to many unpredicted reasons; thus, we should validate the proposed strategy with different initial SOCs to guarantee its robustness.

\subsection{Contribution}

A primary aim of this paper is to present a convenient and effective approach of implementing the A-ECMS for a PHEB running on a fixed route, and the main contribution has the following aspects: (1) A detailed flowchart of PMP algorithm solved by shooting method is presented, and the Secant method is applied to tune the initial co-state value, which makes the shooting operation more efficient and robust even with any properly set initial co-state value. (2) The EF is understood as a major component together with a calibration component, and specifically, the major component defined as a constant plays a dominating role while the effect of the latter including the proportional and integral terms can be greatly reduced, which can ease the tuning of PI coefficients. Based on the PMP solution, the optimal initial co-state value acquired in last shooting is taken as the stable constant of EF. (3) A total of ten successive driving cycles sampled from the bus line are applied to validate the proposed method, in which the first cycle is used as a sample to obtain the EF and other nine cycles to evaluate the A-ECMS. Meanwhile, three initial SOC levels standing for different battery charged condition is set to further show the flexibility of the A-ECMS. 


\subsection{Outline}

The remainder of this paper is organized as follows: Section 2 presents the powertrain of the PHEB and the mathematical model. In Section 3, the PMP is introduced and the shooting method to obtain the numerical solution is also detailed. Then, the adaptive ECMS strategy is proposed in Section 4. After that, the proposed method is examined and compared with the PMP and DP methods in Section 5. Finally, the conclusion is drawn in Section 6.

\section{Model Description}

\subsection{Powertrain Architecture and Model}

\subsubsection{Powertrain Architecture}

The plug-in hybrid electric bus investigated in this paper is designed to serve as a bus in Xi'an City. The powertrain features a serial structure with two power sources including an engine-generator unit (EGU) and two driving motors on both sides, as shown in Figure 1. The particularity of the driveline lies in the two-motor-driving system with each motor connecting to a reducer to drive the wheel respectively, making the powertrain compact to contain more passengers. A natural gas engine mechanically couples to an integrated-stator-generator (ISG) to make up the EGU. The main parameters of the PHEB are summarized in Table 1.

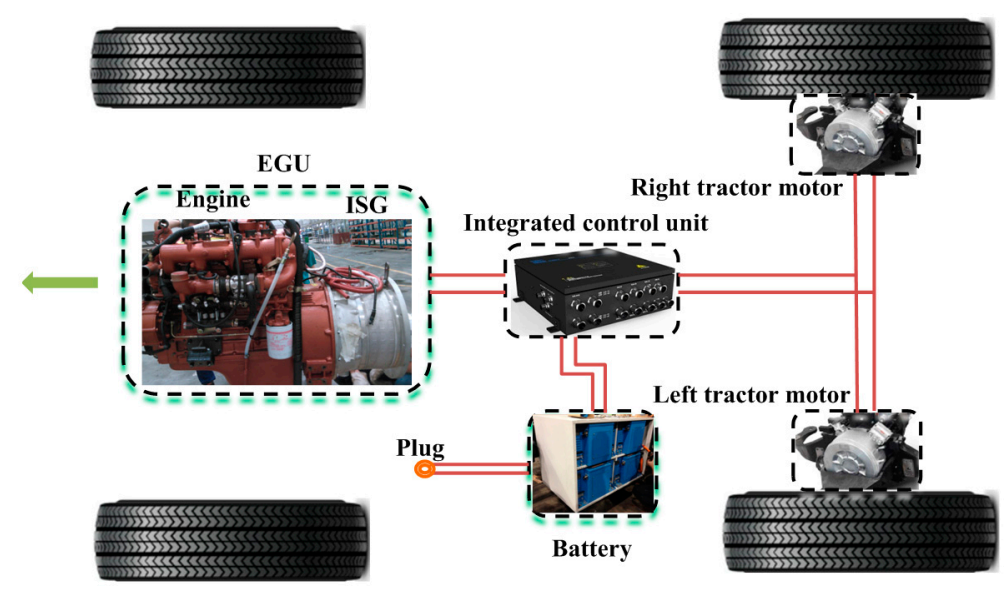

Figure 1. Architecture of the powertrain.

Table 1. The specification of the PHEB.

\begin{tabular}{ccc}
\hline Item & Parameter & Value \\
\hline \multirow{2}{*}{ Vehicle } & Curb weight $(\mathrm{kg})$ & 13,500 \\
& Reducer ratio & 13.9 \\
\hline \multirow{2}{*}{ Engine } & Displacement $(\mathrm{L})$ & 4.2 \\
& Rated power $(\mathrm{kW})$ & 88 \\
& Max speed $(\mathrm{rpm})$ & 2800 \\
\hline \multirow{2}{*}{ ISG } & Max power $(\mathrm{kW})$ & 130 \\
& Max torque $(\mathrm{Nm})$ & 500 \\
\multirow{2}{*}{ Driving motor } & Max speed $(\mathrm{rpm})$ & 6000 \\
& Max power $(\mathrm{kW})$ & 150 \\
& Max torque $(\mathrm{Nm})$ & 650 \\
& Max speed $(\mathrm{rpm})$ & 6000 \\
\hline \multirow{2}{*}{ Battery } & Capacity $(\mathrm{Ah})$ & 120 \\
& Battery total voltage $(\mathrm{V})$ & 537.6 \\
\hline
\end{tabular}




\subsubsection{Powertrain Model}

The quasi-static model is employed to describe the feature of the powertrain.

Motor Model

The tractor machine is the permanent magnet synchronous motor, which can operate in both the motoring and generating mode. The motor efficiency is expressed as the function of the rotational speed and torque, which has the form of:

$$
\eta_{m}=\eta_{m}\left(n_{m}, T_{m}\right)
$$

where $\eta_{m}$ is the motor efficiency, $n_{m}$ and $T_{m}$ are the rotational speed and torque respectively. Based on the experimental data, the efficient map is plotted as the function of the rotational speed and torque, see Figure 2.

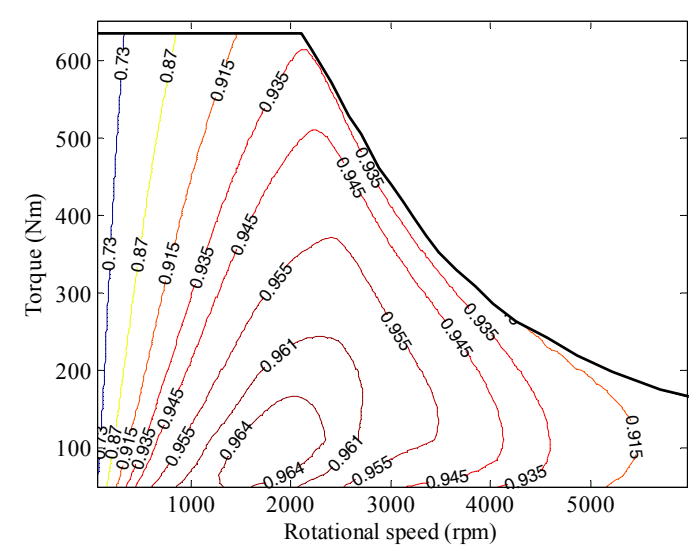

Figure 2. Efficiency map of the driving motor.

EGU Model

The EGU efficiency $\eta_{e g u}$ is also described as the function of its rotational speed $n_{e}$ and torque $T_{e}$ :

$$
\eta_{\text {egu }}=\eta_{\text {egu }}\left(n_{e}, T_{e}\right)
$$

The efficiency map can be figured out by combing the engine BSFC and ISG efficiency together, as shown in Figure 3. According to this map, the optimal fuel rate curve with respect to the output power can be obtained, as plotted in Figure 4.

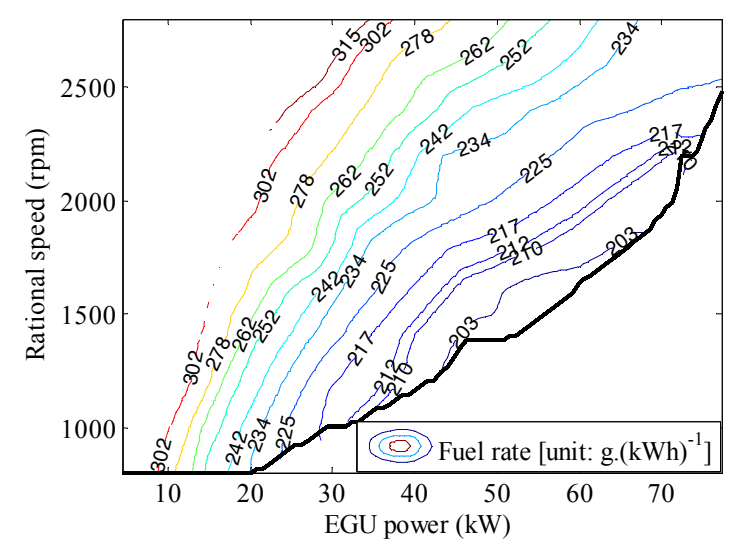

Figure 3. EGU efficiency map. 


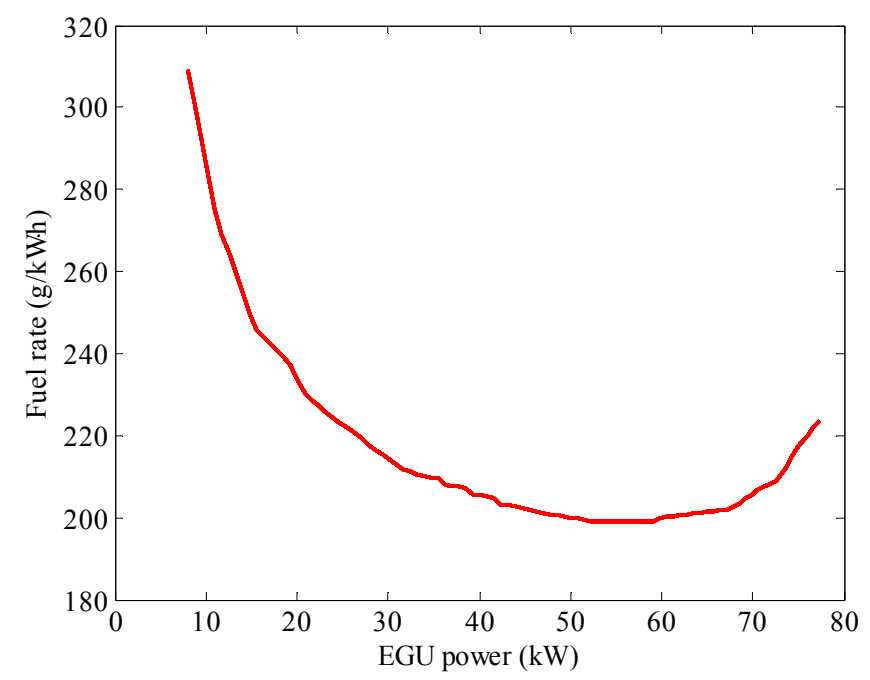

Figure 4. Optimal EGU fuel rate curve.

\subsubsection{Battery Model}

The total voltage of the Li-ion battery pack reaches up to $537.6 \mathrm{~V}$ and the nominal capacity is $120 \mathrm{Ah}$. The Rint model is chosen to simulate the battery dynamics in which the open circuit voltage and internal resistance for the cell battery are described as the function of the SOC [35], as illustrated in Figure 5.

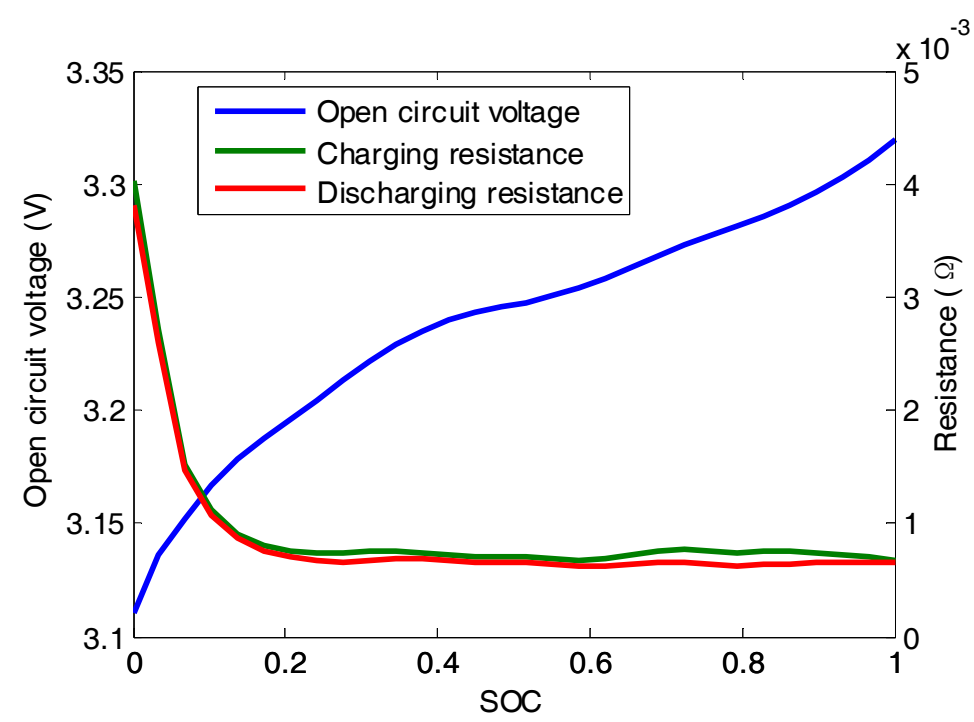

Figure 5. The open circuit voltage and internal resistance with respect to SOC for a cell battery.

Meanwhile, here it is assumed that the cooling and heating system configured for the battery has no impact on the battery power consumption and the power balance equation for the battery system is described as:

$$
P_{b a t}=P_{b}+P_{l}=P_{b}+I R_{b}^{2}
$$

in which $P_{b a t}$ is the total power, $P_{b}$ is the power in and out of the battery, $P_{l}$ is the battery power loss caused by the internal resistance $R_{b}$, and $I$ is the battery current. 
The battery dynamic equation is governed by:

$$
S \dot{S O C}=f(S O C)=-\frac{I}{Q}=-\frac{U_{o c}^{2}-\sqrt{U_{o c}^{2}-4 R_{b} P_{b}}}{2 Q_{b} R_{b}}
$$

where $U_{o c}$ is the open circuit voltage and $Q_{b}$ is the nominal capacity.

\subsection{Longitudinal Dynamics}

The power absorbed by both driving motors is used to overcome the rolling resistance force, air resistance force and accelerating resistance, so the longitudinal dynamics of the bus has the form of:

$$
\begin{gathered}
P_{r}=\frac{2}{\eta_{m}^{\operatorname{sign}\left(T_{m}\right)} \eta_{d}^{\operatorname{sign}\left(T_{m}\right)}} \cdot \frac{T_{m} n_{m}}{9550} \\
T_{m}=\left(\rho m \frac{d v}{d t}+m g f+\frac{C_{d} A v^{2}}{21.15}\right) \frac{r}{i_{g}}
\end{gathered}
$$

where $T_{m}$ and $n_{m}$ are the output torque and rotational speed of each motor; $P_{r}$ is the total power required by the two-motor system; $\eta_{m}$ denotes the motor efficiency and $\eta_{d}$ presents the mechanical efficiency of the driveline; $m$ is the total vehicle mass; $v$ is the speed; $\rho$ is the equivalent rotational inertia; $i_{g}$ is the gear ratio of the two-level reducer; $r$ is the tire radius; $C_{d}$ is the air resistance coefficient; $A$ is the front face area.

Then the electric power balance between the power sources is governed by the following equation:

$$
P_{b}+P_{E G U}=P_{m}+P_{a u x}
$$

in which $P_{E G U}$ is the APU output power, $P_{m}$ is the electric machine power, and $P_{a u x}$ denotes the auxiliary component power, e.g., the electric steering system, the electric braking pump, etc.

\section{Pontryagin's Minimum Principle}

\section{1. $P M P$}

The PMP consists in minimizing the total energy consumption cost during the entire trip, and the objective function can be given as:

$$
J=\min \int_{0}^{t_{f}}\left(c_{f} \dot{m}_{f}+c_{e} \frac{P_{b a t}}{3600}\right) d t
$$

where the instantaneous energy consumption cost contains the fuel cost as well as the electricity cost rather than the only fuel consumption cost so as to yield a general expression to cover all cases even when the electricity price surpasses that of the natural gas.

Accordingly, the Hamilton function can be expressed as [10]:

$$
H=c_{f} \dot{m}_{f}+c_{e} \frac{P_{b a t}}{3600}+\lambda \cdot S \dot{O C C}
$$

in which $\lambda$ is the co-state variable, which evolves with the co-state dynamics [23]:

$$
\dot{\lambda}=-\frac{\partial H}{\partial S O C}=-\lambda \frac{\partial S \dot{O C}}{S O C}
$$


Here the battery SOC is the system state variable and the normal equation has the following formula [23]:

$$
S \dot{C}=\frac{\partial H}{\partial \lambda}=f(S O C)
$$

The constrained boundary values should be respected while minimizing the Hamilton function, that is, for the upper bound:

$$
S O C_{\text {intial }}=S O C_{0}
$$

and for the lower bound:

$$
S O C_{\text {end }}=S O C_{f}
$$

Additionally, the physical limitations imposed by the power components are given as:

$$
\left\{\begin{array}{l}
P_{b a t, \text { min }} \leq P_{b a t} \leq P_{b a t, \max } \\
P_{E G U, \min } \leq P_{E G U} \leq P_{E G U, \max } \\
T_{m, \min } \leq T_{m} \leq T_{m, \max } \\
n_{m, \min } \leq n_{m} \leq n_{m, \max }
\end{array}\right.
$$

Then, the optimal control input will be captured with the expression:

$$
P_{E G U}^{*}=\operatorname{argmin} H\left(S O C^{*}, P_{E G U}, \lambda^{*}, S O C^{*}, t\right)
$$

\subsection{Numerical Solution}

\subsubsection{Shooting Method}

Since it is impossible to solve the PMP with an analytical method, the PMP-based EMS usually can be converted into a two-point boundary value problem (TPBVP), and then obtain the numerical solution by using the shooting method. The most critical step in the shooting method is to tune the co-state, because such a value, if not properly selected, may greatly increase the shooting times or even lead to a failure of shooting process. Therefore, to avoid repeated adjustment of the initial co-state value and make a more efficient search of the optimal co-state value, in this paper, the Secant method is introduced to modify the initial co-state value in each shooting process, which has the form of:

$$
\left\{\begin{array}{c}
\lambda_{1}=\lambda_{0} \quad j=1 \\
\lambda_{2}=\lambda_{0}+\delta \quad j=2 \\
\lambda_{j}=\lambda_{j-1}-\left(\lambda_{j-1}-\lambda_{j-2}\right) \frac{\operatorname{SOC}_{j-1, f}-S_{S O C}}{\operatorname{SOC}_{j-1, f}-S O C_{j-2, f}} j=3,4, \cdots
\end{array}\right.
$$

where $\lambda_{i}$ is the initial co-state value in each shooting; $\lambda_{1}$ and $\lambda_{2}$ are the preset first two initial co-state values determined by the constants $\lambda_{0}$ and $\delta ; S O C_{j, f}$ donates the final SOC in $j$-th shooting operation.

\subsubsection{Algorithm Flowchart}

Figure 6 illustrates the algorithmic flowchart of PMP-based EMS solved by the shooting method in detail, in which the index $i, j$ and $k$ present the $i$-th discrete value of the EGU power, $j$-th shooting sequence and $k$-th step of the driving cycle; $\varepsilon$ is the converging factor to terminate the shooting cycles; and $\Delta P$ denotes the unit increment of the EGU output power. 


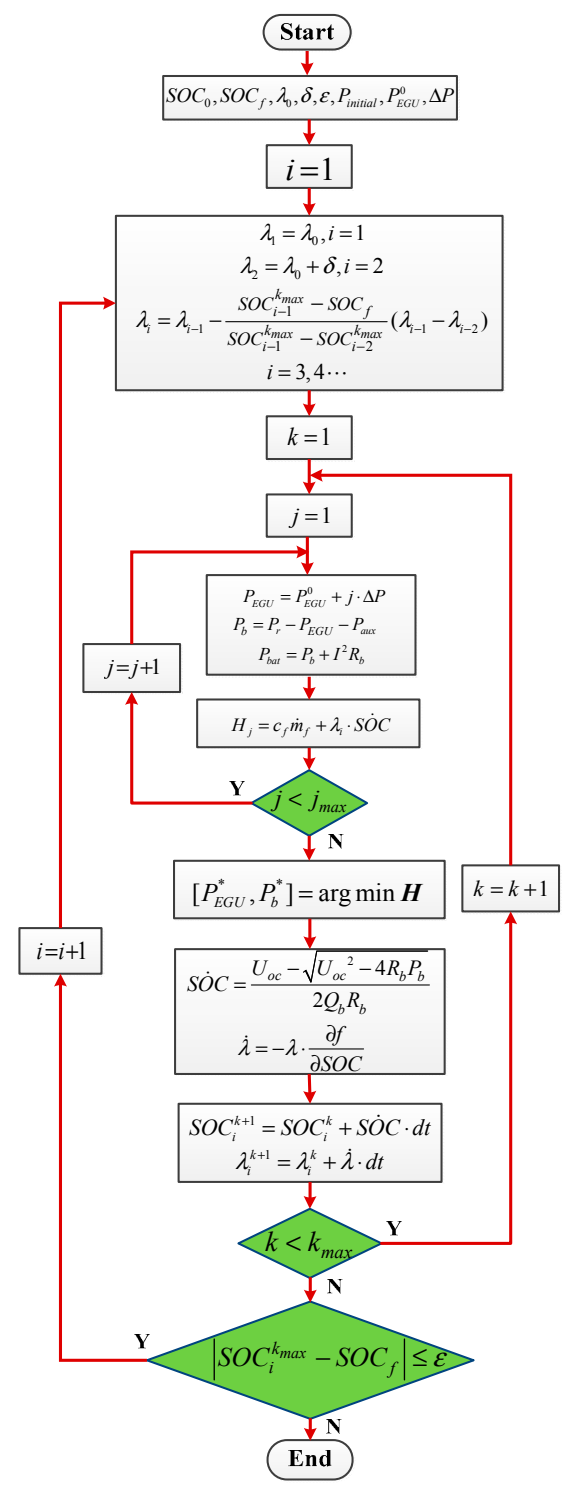

Figure 6. Algorithmic flowchart of the PMP-based EMS solved by the shooting method.

\section{Adaptive ECMS}

\subsection{A-ECMS}

As the ECMS consists in the instantaneous minimum of total equivalent energy consumption cost, then the objective function can be described as:

$$
J=c_{f} \dot{m}_{f}+s(t) \cdot \frac{c_{e} P_{b a t}}{3600}
$$

in which $J$ denotes the total energy consumption cost; $c_{f}$ and $c_{e}$ are the market prices for the natural gas (Yuan $/ \mathrm{m}^{3}$ ) and electricity (Yuan $/ \mathrm{kWh}$ ) respectively, and $s(t)$ is the adaptive EF, which changes dynamically with the driving information.

\subsection{Reference $S O C$}

In order to reach the expected lower boundary SOC at the end of the trip, the reference SOC which is purposely designed to guide the battery to discharge has to be properly defined. Considering 
that the road in $\mathrm{Xi}^{\prime}$ an city is flat enough and the slope is negligible, the reference SOC here is set as the linear function of the current driving distance, which has the form of:

$$
\left\{\begin{array}{l}
S O C_{r e f}(t)=S O C_{\text {initial }}-\frac{D_{\text {now }}(t)}{D_{\text {total }}}\left(S O C_{0}-S O C_{f}\right) \\
D_{\text {now }}(t)=\int_{0}^{t} v(t) d t
\end{array}\right.
$$

where $S O C_{r e f}$ is the reference SOC; $S O C_{\text {inital }}$ and $S O C_{f}$ are the upper and lower SOC boundary values respectively; $D_{\text {total }}$ denotes the average total distance, and $D_{\text {now }}$ presents the travelled distance up to now.

As stated early, the driving distance in most cases are around $70 \mathrm{~km}$, therefore, $D_{\text {total }}$ is set to this value, which reflects the basic information for the fixed bus line. As for the initial SOC which may fluctuate in the daily commute but within a narrow range, thus the proposed controller is examined with three different SOC levels including 0.95, 0.9 and 0.8 to simulate the realistic charging conditions. Since the electricity allows the less expensive propulsion than the fuel, the stored electric energy in battery should be used as much as possible, and the lower boundary value $S O C_{f}$ is set to 0.3 for all cases by considering the battery degradation and longevity.

\subsection{Adaptive Equivalence Factor}

With the adaptive law based on the feedback of battery SOC, the EF is described as a PI controller:

$$
s(t)=s_{0}+s_{v}(t)=s_{0}+k_{p}\left(\operatorname{SOC}_{f}(t)-\operatorname{SOC}(t)\right)+k_{i} \int_{0}^{t}\left(\operatorname{SOC}_{r e f}(t)-\operatorname{SOC}(t)\right) d t
$$

According to Equation (18), three parameters $\left(s_{0}, k_{p}, k_{i}\right)$ exist in the EF which can be divided into two parts: the stable constant or the optimal initial $\mathrm{EF} s_{0}$ and the calibrating component $s_{v}$, including the proportional and integral terms.

\subsection{Optimal Stable Constant of EF}

The battery SOC dynamics can be described as:

$$
f(S O C)=-\frac{I}{Q}=-\frac{I * U_{o c}}{Q * U_{o c}}=-\frac{P_{b a t}}{3.6 E_{b}}
$$

in which $E_{b}$ denotes the battery energy, $\mathrm{KJ}$.

Then the Hamilton function can be rewritten as:

$$
H=c_{f} \dot{m}_{f}+\frac{P_{b a t}}{3600}\left(c_{e}-\frac{1000 \lambda}{E_{b}}\right)
$$

As known, the PMP strategy seeks the optimal power split between the EGU and battery by minimizing the Hamilton function with the SOC boundary constraints, while the ECMS achieves the optimum energy usage cost by dynamically tuning the EF in each time step, and the comparison between the Equations (16) and (20) indicates the identical formula of the instantaneous energy consumption cost. Thus if we let:

$$
c_{e}-\frac{1000 \lambda(t)}{E_{b}}=c_{e} s(t)
$$

then:

$$
s(t)=1-\frac{1000 \lambda(t)}{c_{e} E_{b}}
$$

Equation (22) implies that if the optimal co-state value $\lambda$ is known, then it is capable of capturing the optimal EF $s$ in ECMS. Further, since the optimal co-state trace unveils the optimal power allocation relationship, thus the initial co-state value in last shooting can be taken as an ideal stable constant of 
$\mathrm{EF}$, which will guide the SOC to change along the reference trajectory and acquire the similar solution as that of the PMP algorithm.

Overall, the similarity between the presented A-ECMS and PMP method lines in two aspects. Firstly, the instantaneous minimum of the Hamilton function is equivalent to the expression of ECMS in Equation (16); as for the SOC boundary constraints imposed by PMP algorithm, the PI terms are supplemented in A-ECMS to guarantee the SOC to follow the reference trajectory, which is generated with the aid of route distance.

Based on Equation (22), the optimal stable constant of $\mathrm{EF} s_{0, o p t}$ can be rewritten as:

$$
s_{0, o p t}=1-\frac{1000 \lambda_{0, o p t}}{c_{e} E_{b}}
$$

where $\lambda_{0, \text { opt }}$ denotes the optimal initial co-state value obtained in last shooting operation.

\subsection{Scheme of A-ECMS}

Figure 7 illustrates the scheme of the proposed A-ECMS for the PHEB. The optimal constant of EF is firstly calculated by using the PMP algorithm based on the sample driving cycle. Then, the SOC feedback can be calculated with the reference SOC, in which the route information is utilized. After that, it is able to obtain the EF by modifying the PI terms to achieve the power distribution policy.

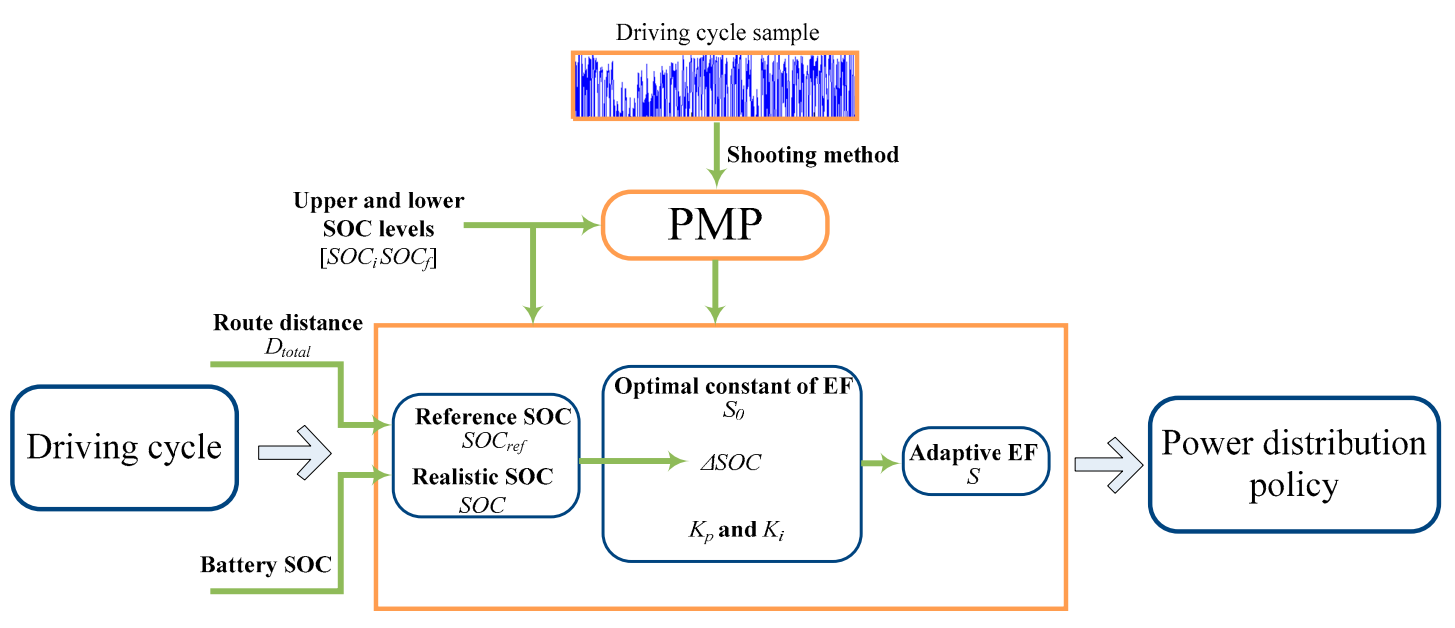

Figure 7. Scheme of proposed A-ECMS.

\section{Validation and Discussion}

\subsection{Route Discription}

To validate the proposed methodology, a bus route in Xi'an City was selected. It features a round trip (go-and-return path), and the collected speed profiles for nearly five months were available. Although the driving cycle in regular commuting is unknown in advance, the long-term record of the speeding profiles can still provide valuable information. The statistics demonstrates the regular driving distance on the fixed line always maintains around $70.0 \mathrm{~km}$, which can offer the critical parameter to generate the reference SOC. Moreover, while the daily speeding profiles are seemingly different from each other, they still exhibit an internal consistency, owing to certain constant factors, such as the vehicle population, inhabitant trip characteristics, fixed stopping stations, road intersection and traffic signal control system. Therefore, each speeding profile that basically reflects the environmental character of this route can be viewed as a representative of the daily driving cycles. 


\subsection{Calculation of Optimal Constant of EF}

Without the loss of the generality, ten successive speeding profiles (numbered from 1 to 10) are selected from the historical data for the bus line to evaluate the proposed method, see Figure 8. And the first profile is used as a sample to compute the optimal initial co-state in the PMP strategy and then the optimal stable constant of EF, while the other nine samples are employed to validate the A-ECMS performance. Figure 9 summaries the driving distance and time for these cycles and the No. 1 cycle reaches $67.16 \mathrm{~km}$ and lasts $5.39 \mathrm{~h}$.

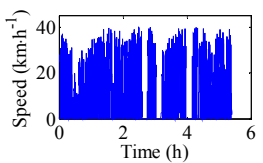

No. 1

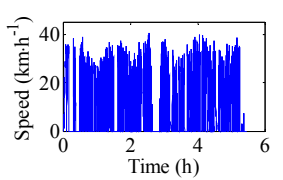

No. 6

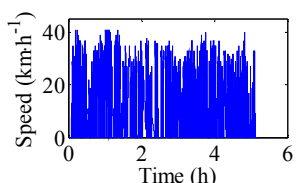

No. 2

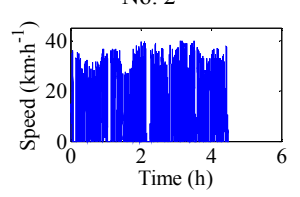

No. 7

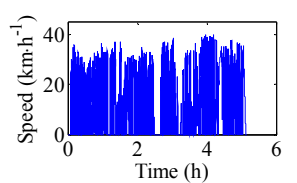

No. 3

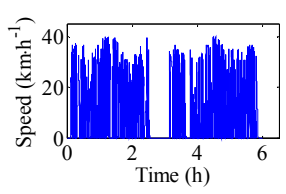

No. 8

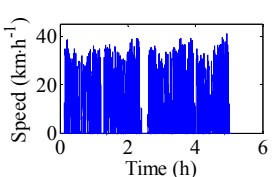

No. 4

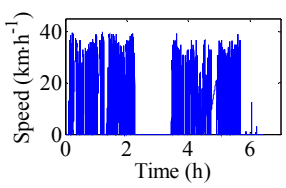

No. 9
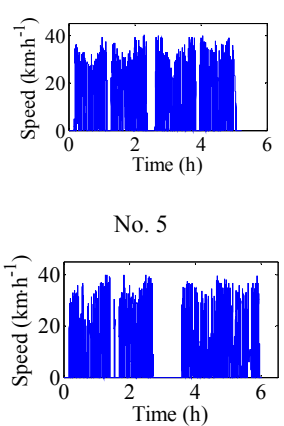

No. 10

Figure 8. Ten successive speeding profiles selected from the historical data.

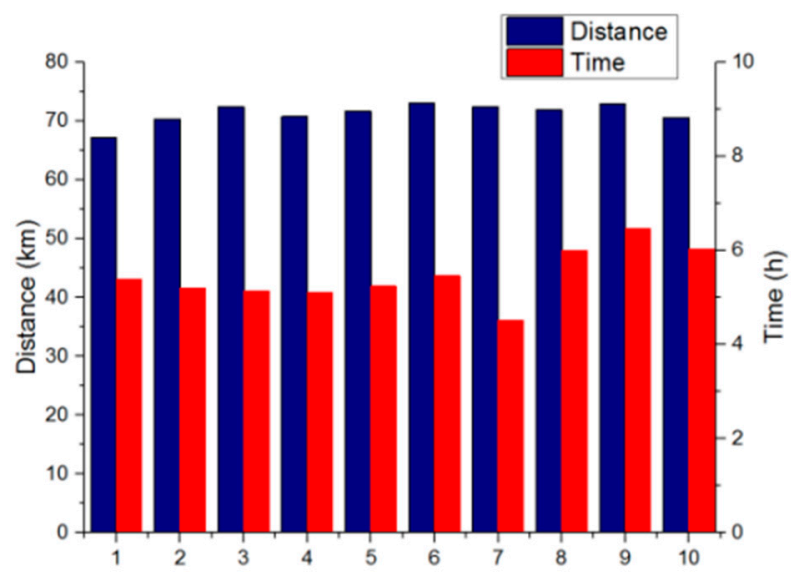

Figure 9. Histogram of the driving distance and time for the 10 driving cycles.

Meanwhile, as a typical sample to compute the stable constant of EF, the initial battery SOC in the daily trip should be taken into account and here the initial SOC is set to 0.9 to simulate a fully charged battery. In order to underline the robustness of the shooting method, the initial co-state is set to three different values $\left(\lambda_{0}=-20,-15\right.$, and -10 respectively). Besides, the parameter $\delta$ is given as -1.0 , and the convergence factor $\varepsilon$ that affects the shooting times is set to 0.001 . All the relevant parameters in the PMP algorithm are listed in Table 2. The corresponding SOC traces and the initial co-state values acquired by the shooting method are plotted in Figures 10-12. In case 1, four SOC curves are generated, meaning four shootings are needed to hit the target value, and the initial co-state value adjusted by the Secant method continuously undergoes rises and falls before arriving at the end value; Six shootings are required in case 2 to meet the convergence condition, and the initial co-state value increases successively; as for case 3 , the initial co-state, however, declines gradually during the four shooting operations. 


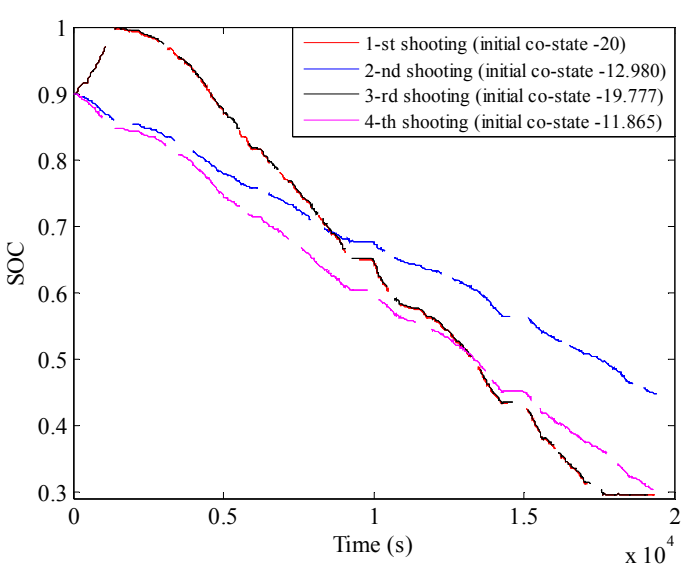

(a)

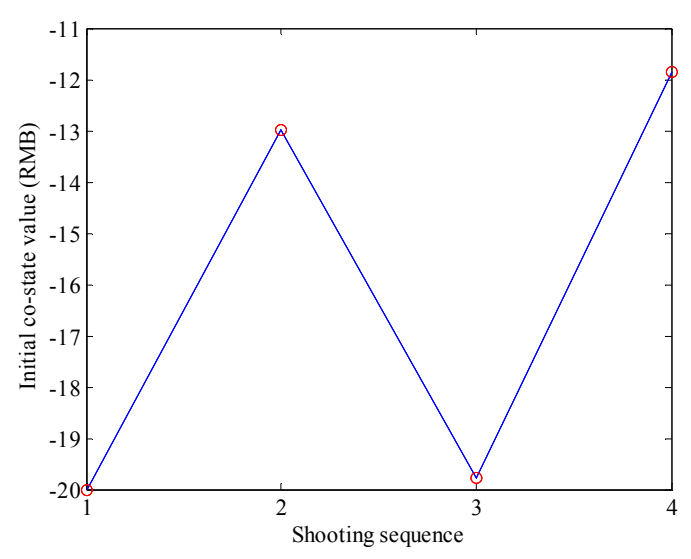

(b)

Figure 10. Results of $\lambda_{0}=-20$ (a) SOC profiles; (b) Initial co-state values during the shooting process.

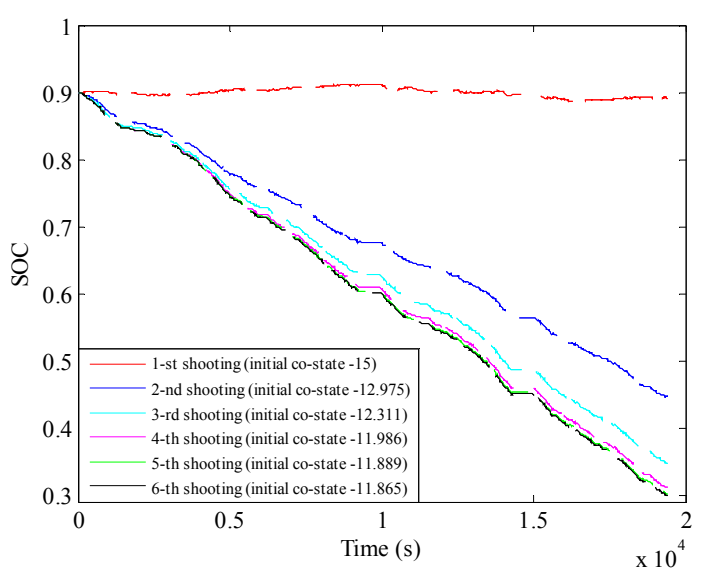

(a)

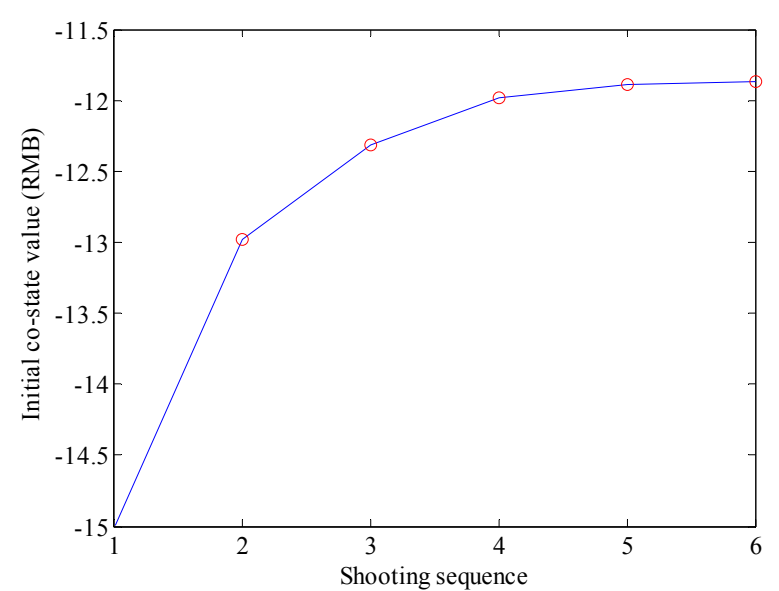

(b)

Figure 11. Results of $\lambda_{0}=-15$ (a) SOC profiles; (b) Initial co-state values during the shooting process.

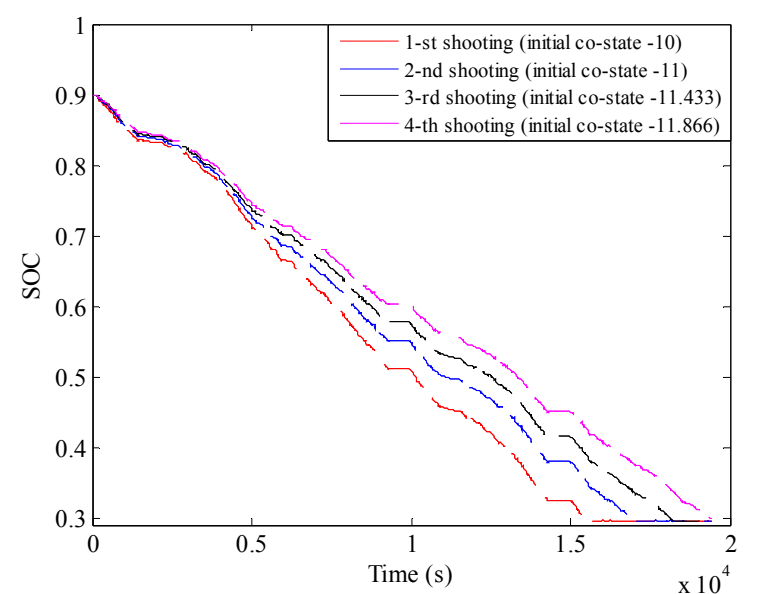

(a)

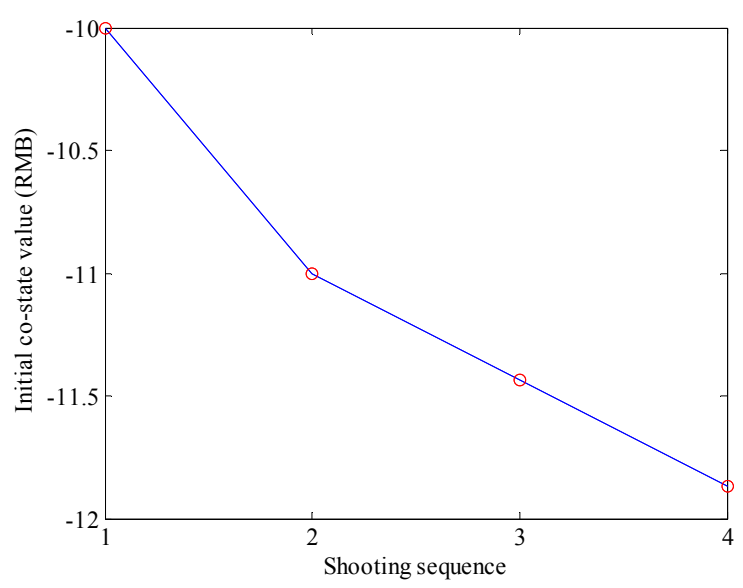

(b)

Figure 12. Results of $\lambda_{0}=-10$ (a) SOC profiles; (b) Initial co-state values during the shooting process. 
Table 2. Configuration of the parameters.

\begin{tabular}{cccccc}
\hline Parameter & Value & Parameter & Value & Parameter & Value \\
\hline$c_{f}\left(\right.$ Yuan $\left.\cdot \mathrm{m}^{-3}\right)$ & 3.7 & SOC upper level & 0.9 & $\lambda_{0}$ & $-20 /-15 /-10$ \\
$c_{e}\left(\right.$ Yuan $\left.\cdot \mathrm{kWh} \mathrm{h}^{-1}\right)$ & 0.8 & SOC lower level & 0.3 & $\delta$ & -1.0 \\
$\Delta P(\mathrm{~kW})$ & 0.5 & $d t(\mathrm{~s})$ & 1 & $\varepsilon$ & 0.001 \\
\hline
\end{tabular}

The results in Figures 10-12 show that in spite of different initial co-state values set in the beginning, the optimal initial co-state value obtained in last shooting sequence almost reached the same end value ( -11.865 for $\lambda_{0}=-20$ and $-10,-11.866$ for $\left.\lambda_{0}=-15\right)$, indicating the robustness of the shooting method. Accordingly, the optimal co-state trace yielded in the last shooting operation is illustrated in Figure 13, which reveals the essence of power allocation relationship between the EGU and battery. Based on Equation (23), the optimal stable constant of EF $s_{0}$ (1.2299) can be figured out with the optimal initial co-state value $(-11.865)$.

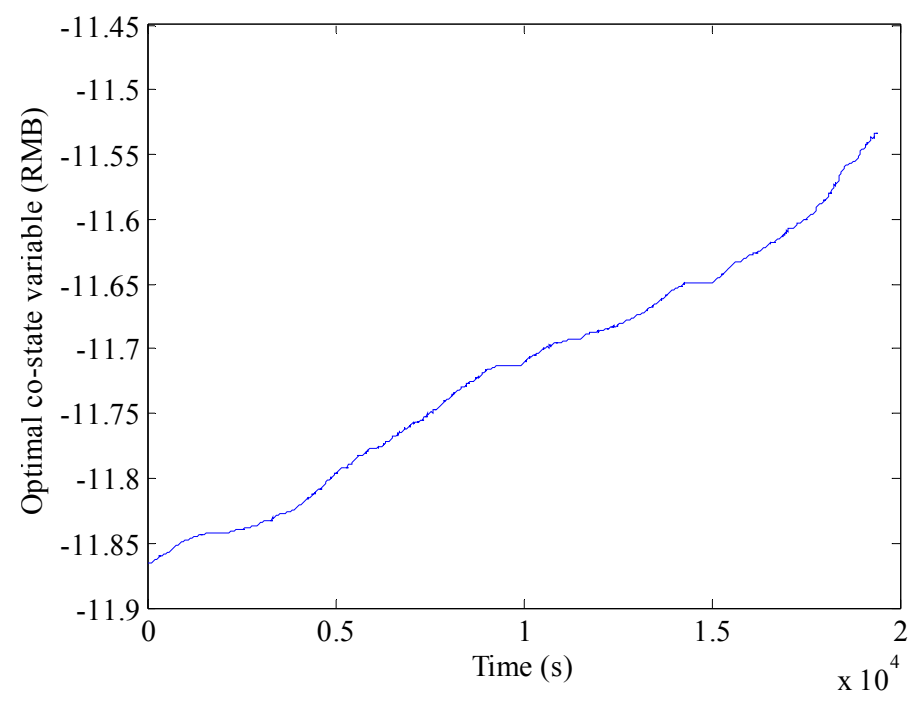

Figure 13. Optimal co-state variable trajectory.

\subsection{Validation of A-ECMS}

The driving cycles (from No. 2 to 10) are applied to examine the proposed A-ECMS. In addition, the initial battery SOC at the starting station does not always hold exactly the same level, due to many unpredictable reasons and random factors. So, in order to cover possible conditions, the initial SOC is set to three levels ranging from $0.95-0.80$, including the fully charged battery $\left(S O C_{0}=0.95\right)$, the moderately fully charged battery $\left(S O C_{0}=0.90\right)$, and the special case $\left(S O C_{0}=0.80\right)$, i.e., the bus may undergo some extra trip and consume the electricity before the starting departure. Besides, since all the ten driving distances ranging from 67.1 to $73.1 \mathrm{~km}$ holds around $70 \mathrm{~km}$, therefore, the total distance is set to $70 \mathrm{~km}$ to yield the reference SOC. In addition, the duration of these trips varies with the minimum $4.4 \mathrm{~h}$ and maximum $6.4 \mathrm{~h}$, mainly due to the different stopping time on the final station.

With the given parameters, Equation (23) can be expressed as:

$$
s_{0, o p t}=1-\frac{1000 \lambda_{0, o p t}}{0.8 \times 120 \times 537.6}=1-0.0194 \lambda_{0, o p t}
$$

Multiplied by a relative small constant (0.0194), the second term in Equation (24) will yield a slight variation when the optimal initial co-state $\lambda_{0, \text { opt }}$ changes. As a result, though the No. 1 cycle is randomly chosen as the sample to compute $s_{0, o p t}$, the generality will hold true for all other cases, 
and the only difference between them is the slight change of the optimal stable constant, and thus the subtle calibration of the PI coefficients $\left(k_{p}\right.$ and $k_{i}$ ) accordingly.

With different initial co-state values, Table 3 summaries the optimal initial co-state values, and the corresponding optimal $\mathrm{EF} s_{0, o p t}$. It can be evidently observed that three initial co-state values in each case almost generate the same optimal co-state values; moreover, the optimal co-state value among different cases, denoted as their average values $\bar{\lambda}_{0, o p t}$, varies within a tight interval $[-12.218,-11.846]$, leading to a smaller variation of $s_{0, o p t}$ ranging from [1.2295, 1.2367] according to Equation (24). Therefore, it can be concluded that the optimal stable constant of EF for daily speeding profiles always keep in a narrow range, and the reason attributes to some constant influencing factors including the vehicle population, inhabitants trip characteristics, fixed stopping stations and road intersections, as well as the traffic signal control system. And on the other hand, the proportional and integral terms which is originally devised to correct the $\mathrm{EF}$, can further be adjusted to compensate the variation of $\mathrm{EF}$, so as to achieve an improved fuel economy.

Table 3. Optimal co-state value and optimal stable constant of EF.

\begin{tabular}{|c|c|c|c|c|c|}
\hline \multirow{2}{*}{ Cycle Number } & \multicolumn{3}{|c|}{$\lambda_{0, o p t}$} & \multirow{2}{*}{$\begin{array}{l}- \\
\lambda_{0, o p t}\end{array}$} & \multirow{2}{*}{$s_{0, o p t}$} \\
\hline & $\lambda_{0}=-20$ & $\lambda_{0}=-15$ & $\lambda_{0}=-10$ & & \\
\hline 1 & -11.865 & -11.866 & -11.865 & -11.865 & 1.2299 \\
\hline 2 & -11.909 & -11.909 & -11.909 & -11.909 & 1.2308 \\
\hline 3 & -12.218 & -12.217 & -12.218 & -12.218 & 1.2367 \\
\hline 4 & -12.049 & -12.045 & -12.047 & -12.047 & 1.2334 \\
\hline 5 & -11.941 & -11.940 & -11.940 & -11.940 & 1.2314 \\
\hline 6 & -12.001 & -11.996 & -12.000 & -11.999 & 1.2325 \\
\hline 7 & -11.846 & -11.847 & -11.846 & -11.846 & 1.2295 \\
\hline 8 & -11.979 & -11.975 & -11.975 & -11.976 & 1.2321 \\
\hline 9 & -12.101 & -12.101 & -12.102 & -12.101 & 1.2345 \\
\hline 10 & -11.953 & -11.953 & -11.956 & -11.954 & 1.2316 \\
\hline
\end{tabular}

Compared to the tuning of three coefficients $\left(s_{0}, k_{p}\right.$ and $\left.k_{i}\right)$ simultaneously, it turns out to be very simple to calibrate the two PI coefficients $\left(k_{p}\right.$ and $\left.k_{i}\right)$ with the known optimal constant $s_{0}$, because the combination of the proportional and integral terms, which is to adapt to the disparity between daily speeding profiles, almost accounts for a small proportion of EF. After a few trials, a satisfactory consequence can be obtained with $k_{p}=0.001$ and $k_{i}=0.0008$.

Apart from the PMP algorithm, the DP method is also conducted to compare with the A-ECMS. Based on Bellman's principle, the discrete DP formula can be described as:

For $k=k_{\text {max }}$ :

$$
J_{k}\left(S O C_{h}\right)=\min _{i \in I} g\left(P_{E G U, i}, S O C_{h}\right)
$$

and for $k=k_{\max }-1, k_{\max }-2, \cdots, 1$

$$
J_{k}\left(S_{S O C}\right)=\min _{i \in I}\left\{g\left(P_{E G U, i}, S O C_{h}\right)+J_{k+1}\left(f\left(P_{E G U, i}, S O C_{h}\right)\right)\right\}
$$

In which $k$ is the time step; index $h$ and $i$ denote the $h$-th and $i$-th discrete points of the EGU power and SOC in the allowable sets respectively; $g(\cdot)$ is the instantaneous energy consumption cost, $f(\cdot)$ is the state equation and $J(\cdot)$ is the accumulative energy consumption cost.

The numerical solution of the DP algorithm is greatly related to the number of discrete input and state points as well as the interpolation method to estimate the cost-to-go value. After several trails, both the input and state variables are specified to 200 points, and the linear interpolation is employed to carry out the interpolation calculation.

Figures 14-23 present the SOC profiles for the ten cycles with three initial SOC levels. It can be seen that the battery SOC yielded by the proposed A-ECMS traces the reference value well for all 
cases, and the best results appear in case 8 where the three SOC trajectories almost overlap with the reference curves. In case 6 and case 7 , there are certain disparity between two SOC curves, and more specifically, the SOC surpasses the reference value in the first half trip, and thereafter, the SOC is below the expected reference line; while during the last period of the trip, the surpassing happens again until the end. Despite of that, the total energy consumption cost is almost close to both the DP and PMP results in all cases, owing to the same SOC levels as well as the identical gas usage at the end of the cycle, as can be seen in Tables 4-6.

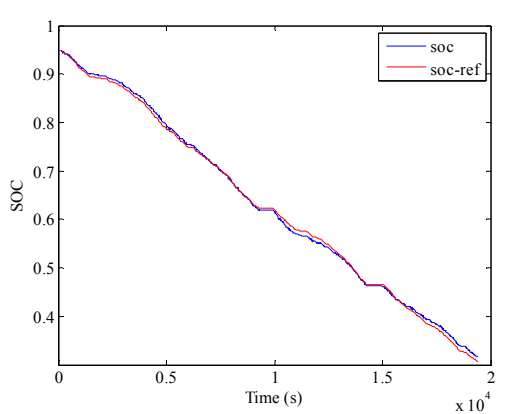

(a)

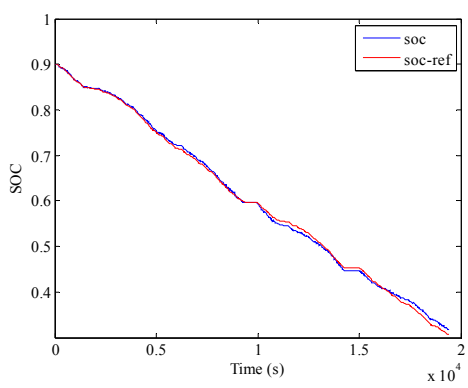

(b)

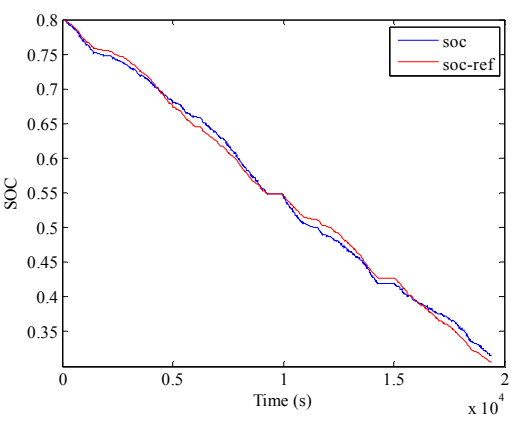

(c)

Figure 14. Comparison of $S O C$ profiles for No. 1 cycle: (a) $S O C_{0}=0.95 ;$ (b) $S O C_{0}=0.9$; (c) $S O C_{0}=0.8$.

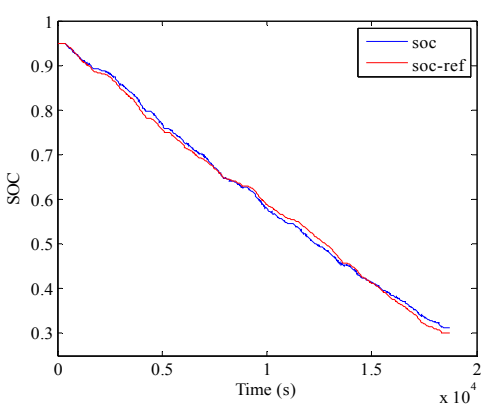

(a)

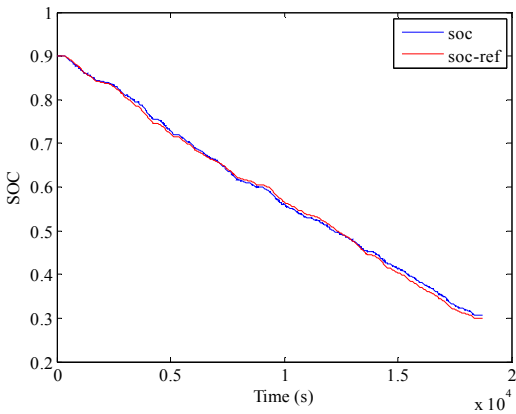

(b)

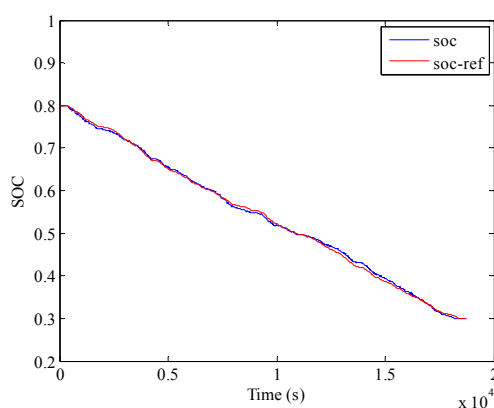

(c)

Figure 15. Comparison of SOC profiles for No. 2 cycle: (a) $S O C_{0}=0.95 ;$ (b) $S O C_{0}=0.9 ;$ (c) $S O C_{0}=0.8$.

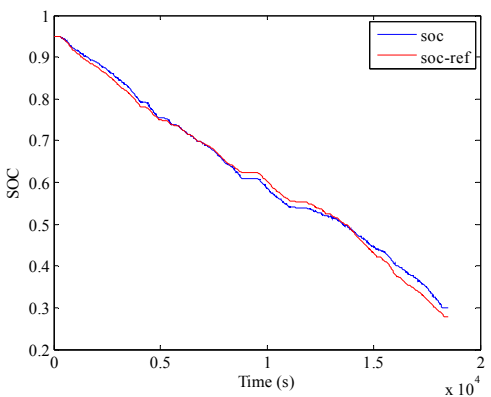

(a)

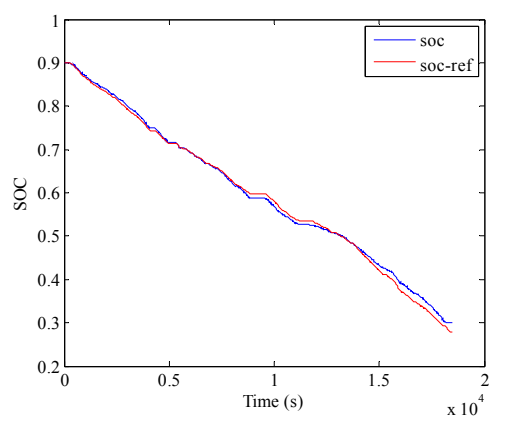

(b)

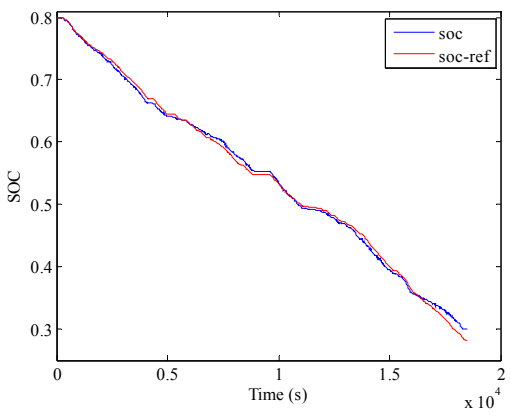

(c)

Figure 16. Comparison of SOC profiles for No. 3 cycle: (a) $S O C_{0}=0.95 ;$ (b) $S O C_{0}=0.9 ;$ (c) $S O C_{0}=0.8$. 


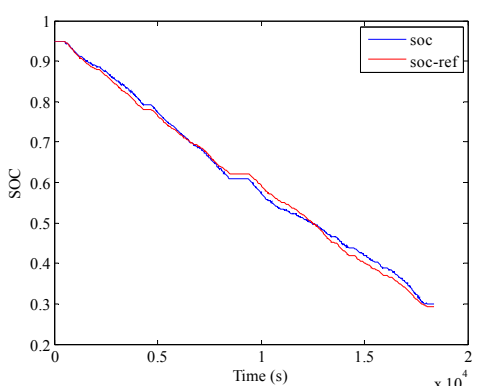

(a)

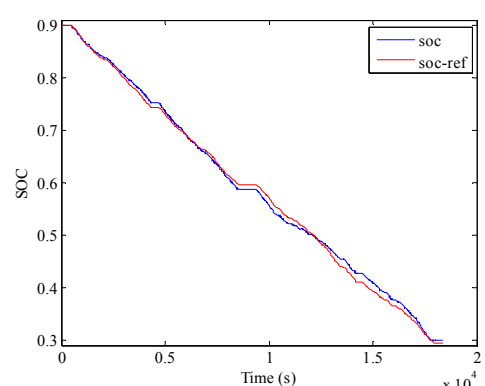

(b)

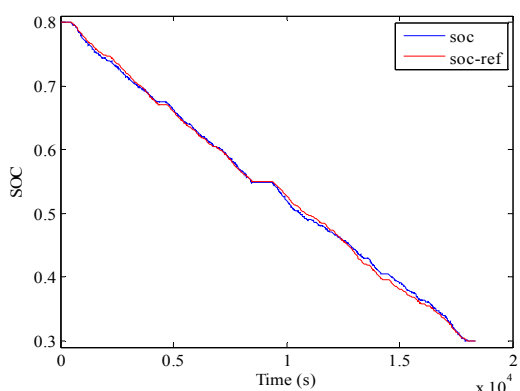

(c)

Figure 17. Comparison of SOC profiles for No. 4 cycle: (a) $S O C_{0}=0.95 ;$ (b) $S O C_{0}=0.9 ;$ (c) $S O C_{0}=0.8$.

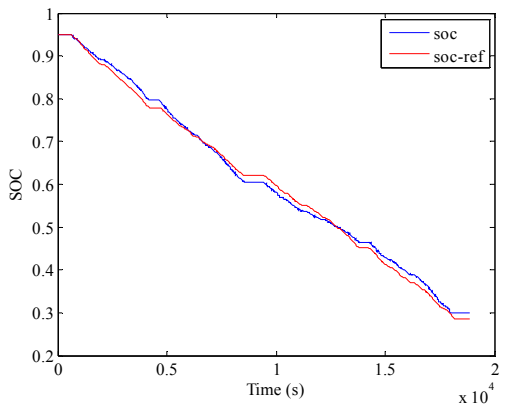

(a)

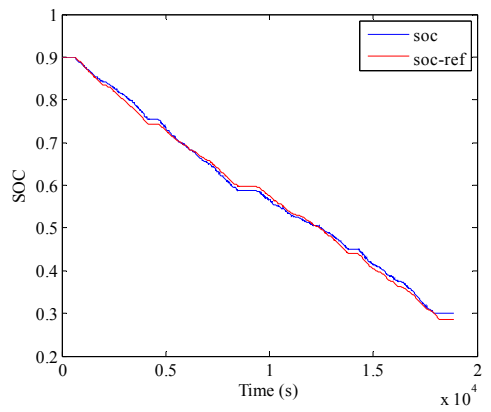

(b)

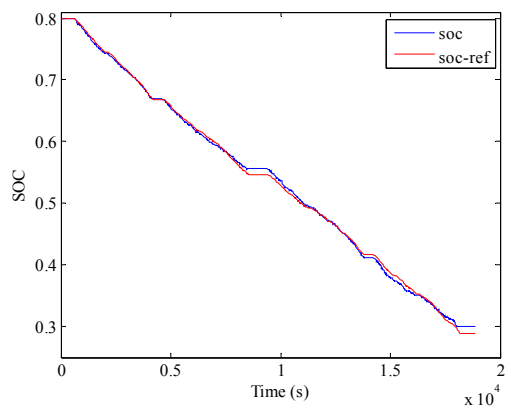

(c)

Figure 18. Comparison of $S O C$ profiles for No. 5 cycle: (a) $S O C_{0}=0.95 ;$ (b) $S O C_{0}=0.9 ;$ (c) $S O C_{0}=0.8$.

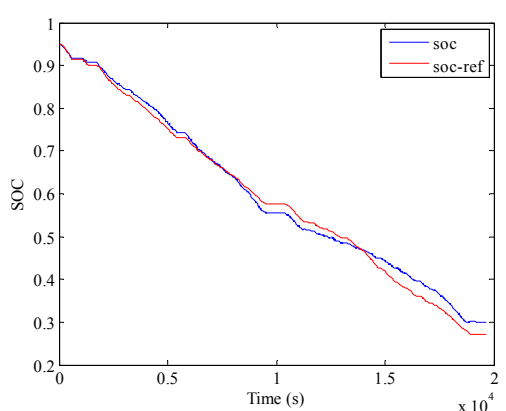

(a)

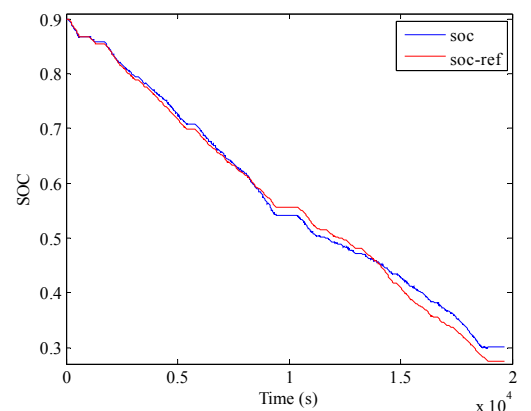

(b)

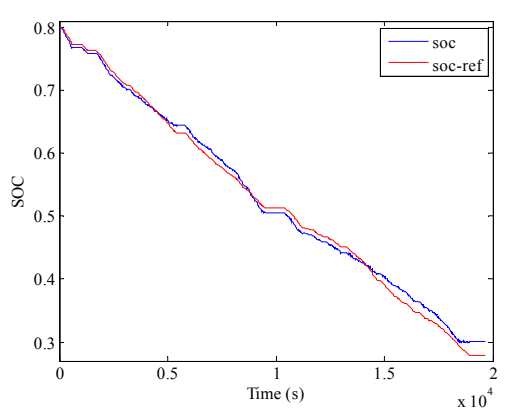

(c)

Figure 19. Comparison of SOC profiles for No. 6 cycle: (a) $S O C_{0}=0.95 ;(\mathbf{b}) S O C_{0}=0.9 ;$ (c) $S O C_{0}=0.8$.

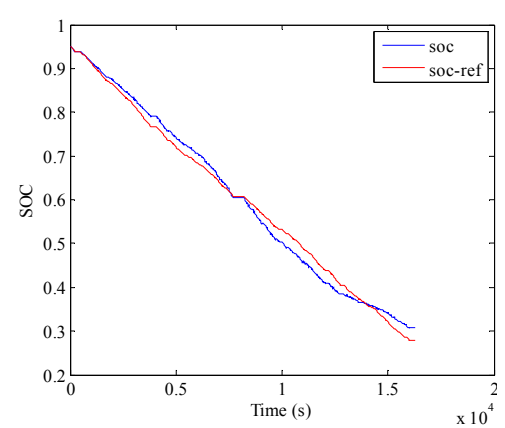

(a)

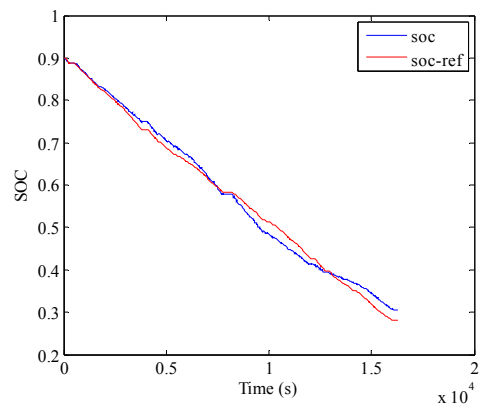

(b)

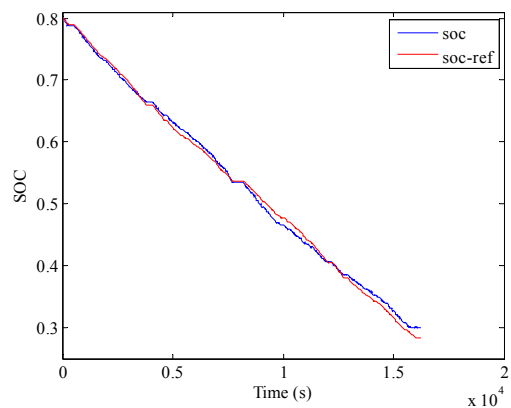

(c)

Figure 20. Comparison of SOC profiles for No. 7 cycle: (a) $S O C_{0}=0.95$; (b) $S O C_{0}=0.9$; (c) $S O C_{0}=0.8$. 


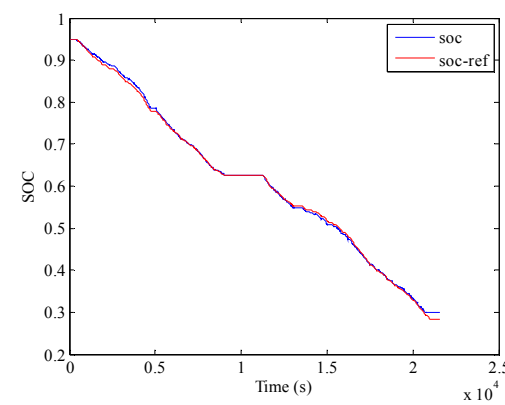

(a)

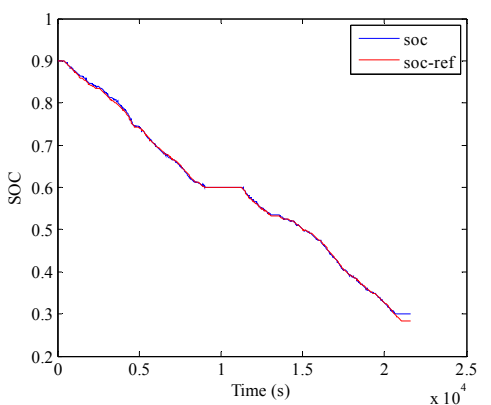

(b)

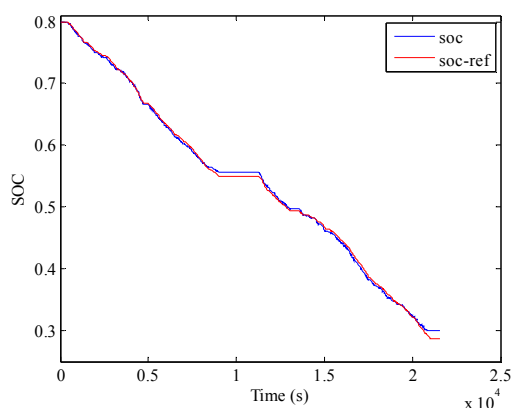

(c)

Figure 21. Comparison of SOC profiles for No. 8 cycle: (a) $S O C_{0}=0.95 ;$ (b) $S O C_{0}=0.9$; (c) $S O C_{0}=0.8$.

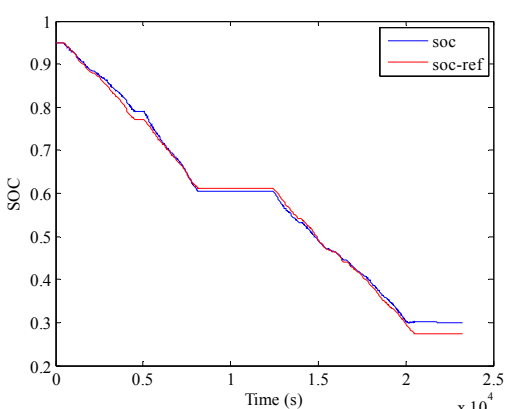

(a)

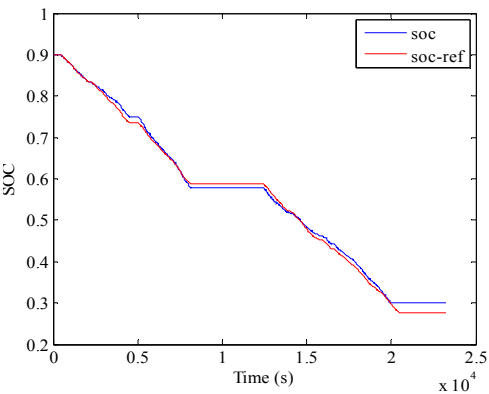

(b)

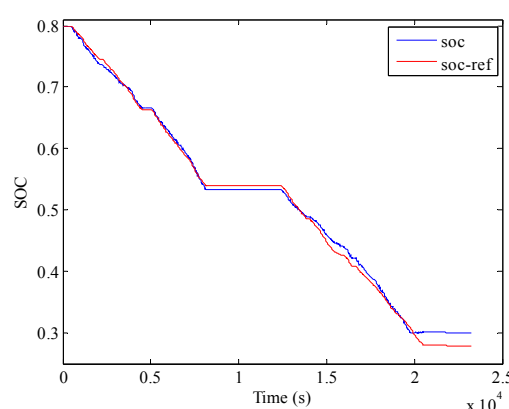

(c)

Figure 22. Comparison of SOC profiles for No. 9 cycle: (a) $S O C_{0}=0.95 ;$ (b) $S O C_{0}=0.9 ;$ (c) $S O C_{0}=0.8$.

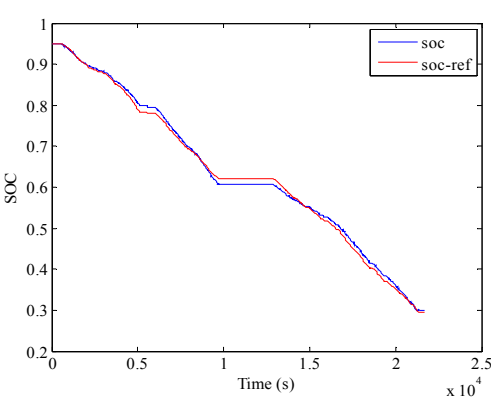

(a)

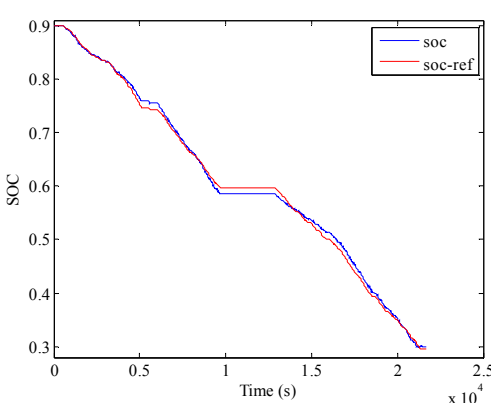

(b)

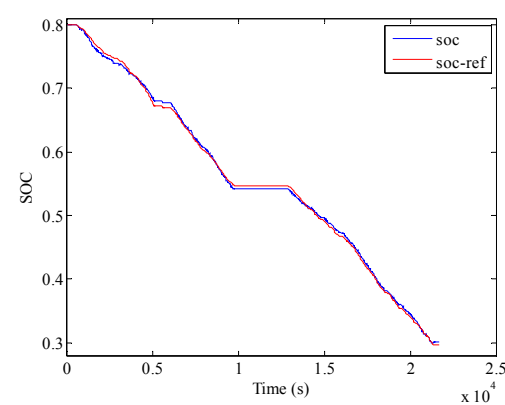

(c)

Figure 23. Comparison of SOC profiles for No. 10 cycle: (a) $S O C_{0}=0.95 ;(\mathbf{b}) S O C_{0}=0.9$; (c) $S O C_{0}=0.8$.

Table 4. Results of the A-ECMS with $S O C_{0}=0.95$.

\begin{tabular}{|c|c|c|c|c|c|c|}
\hline \multirow{2}{*}{$\begin{array}{l}\text { Cycle } \\
\text { Number }\end{array}$} & \multirow{2}{*}{$\begin{array}{c}\text { Gas } \\
\text { Consumption } \\
\left(\mathrm{m}^{3}\right)\end{array}$} & \multirow{2}{*}{$\begin{array}{c}\text { Electricity } \\
\text { Consumption } \\
(\mathrm{kWh})\end{array}$} & \multirow[t]{2}{*}{ Final SOC } & \multicolumn{3}{|c|}{$\begin{array}{c}\text { Total Energy Consumption Cost } \\
\text { (Yuan) }\end{array}$} \\
\hline & & & & A-ECMS & PMP & DP \\
\hline 1 & 3.79 & 41.96 & 0.317 & 47.60 & 47.40 & 47.87 \\
\hline 2 & 3.35 & 42.19 & 0.313 & 46.14 & 45.97 & 46.59 \\
\hline 3 & 3.71 & 43.51 & 0.300 & 48.54 & 48.57 & 49.26 \\
\hline 4 & 3.90 & 43.42 & 0.301 & 49.16 & 49.17 & 49.55 \\
\hline 5 & 3.50 & 44.02 & 0.300 & 48.16 & 48.28 & 48.83 \\
\hline 6 & 3.58 & 43.77 & 0.301 & 48.28 & 48.29 & 48.71 \\
\hline 7 & 4.03 & 42.57 & 0.308 & 48.99 & 48.76 & 49.18 \\
\hline 8 & 3.39 & 43.88 & 0.300 & 47.66 & 47.79 & 48.42 \\
\hline 9 & 3.84 & 44.45 & 0.301 & 49.78 & 49.99 & 50.34 \\
\hline 10 & 3.57 & 43.55 & 0.300 & 48.04 & 48.08 & 48.47 \\
\hline
\end{tabular}


Table 5. Results of the A-ECMS with $S O C_{0}=0.9$.

\begin{tabular}{ccccccc}
\hline $\begin{array}{c}\text { Cycle } \\
\text { Number }\end{array}$ & $\begin{array}{c}\text { Gas } \\
\text { Consumption } \\
\mathbf{( m}^{\mathbf{3}} \mathbf{)}\end{array}$ & $\begin{array}{c}\text { Electricity } \\
\text { Consumption } \\
\mathbf{( k W h )}\end{array}$ & Final SOC & \multicolumn{3}{c}{$\begin{array}{c}\text { Total Energy Consumption Cost } \\
\text { (Yuan) }\end{array}$} \\
\cline { 5 - 7 } & 4.69 & 38.57 & 0.318 & 48.21 & 47.99 & 48.42 \\
1 & 4.11 & 39.31 & 0.306 & 46.64 & 46.56 & 47.11 \\
2 & 4.47 & 40.62 & 0.300 & 49.04 & 49.18 & 49.83 \\
3 & 4.68 & 40.43 & 0.301 & 49.68 & 49.78 & 50.12 \\
4 & 4.27 & 41.05 & 0.300 & 48.66 & 48.87 & 49.41 \\
5 & 4.38 & 40.69 & 0.301 & 48.77 & 48.89 & 49.27 \\
6 & 4.88 & 39.32 & 0.306 & 49.51 & 49.35 & 49.74 \\
7 & 4.22 & 40.73 & 0.300 & 48.22 & 48.39 & 48.97 \\
8 & 4.62 & 41.51 & 0.301 & 50.30 & 50.59 & 50.92 \\
9 & 4.37 & 40.50 & 0.300 & 48.57 & 48.68 & 49.04 \\
\hline 10 & & & & & & A-ECMS \\
\hline
\end{tabular}

Table 6. Results of the A-ECMS with $S O C_{0}=0.8$.

\begin{tabular}{|c|c|c|c|c|c|c|}
\hline \multirow[t]{2}{*}{$\begin{array}{l}\text { Cycle } \\
\text { Number }\end{array}$} & \multirow{2}{*}{$\begin{array}{c}\text { Gas } \\
\text { Consumpiton } \\
\left(\mathrm{m}^{3}\right)\end{array}$} & \multirow{2}{*}{$\begin{array}{c}\text { Electricity } \\
\text { Consumption } \\
(\mathrm{kWh})\end{array}$} & \multirow[t]{2}{*}{ Final SOC } & \multicolumn{3}{|c|}{$\begin{array}{c}\text { Total Energy Consumption Cost } \\
\text { (Yuan) }\end{array}$} \\
\hline & & & & A-ECMS & PMP & DP \\
\hline 1 & 6.44 & 32.01 & 0.32 & 49.45 & 49.23 & 49.62 \\
\hline 2 & 5.65 & 33.54 & 0.30 & 47.72 & 47.81 & 48.33 \\
\hline 3 & 6.42 & 33.30 & 0.30 & 50.41 & 50.44 & 51.10 \\
\hline 4 & 6.48 & 33.67 & 0.30 & 50.92 & 51.03 & 51.35 \\
\hline 5 & 6.17 & 33.92 & 0.30 & 49.96 & 50.12 & 50.61 \\
\hline 6 & 6.06 & 34.38 & 0.30 & 49.92 & 50.14 & 50.52 \\
\hline 7 & 6.37 & 33.67 & 0.30 & 50.49 & 50.59 & 50.97 \\
\hline 8 & 6.12 & 33.61 & 0.30 & 49.54 & 49.63 & 50.21 \\
\hline 9 & 6.31 & 35.15 & 0.30 & 51.48 & 51.85 & 52.16 \\
\hline 10 & 6.25 & 33.41 & 0.30 & 49.86 & 49.92 & 50.27 \\
\hline
\end{tabular}

Despite the three initial SOCs set in purpose, the noticeable differences cannot be observed between these cases. Moreover, although the optimal initial co-state is computed by the shooting method with $S O C_{0}=0.9$, the superiority of the results for this charged level can be negligible, indicating the flexibility of the A-ECMS. Meanwhile, a clear platform stage can be noticed in these SOC curves where the SOC remain unchanged, which attributes to a period of stopping time at the final station.

Compared with the global optimal methods, the proposed A-ECMS achieves the similar total energy consumption cost in all cases with three initial SOCs, indicating the adaptability of this method. Besides, the total cost of PMP method is always less than that of DP algorithm, and the reason is that the DP has to perform the iterative calculation with massive discrete state and input variables as well as execute the interpolation method to estimate the cost-to-go value, which will somewhat weaken the solution precision; as for the PMP algorithm, however, the accuracy mainly depends on the convergence factor and presents a superior performance in terms of time usage and numerical solution.

The traces of the adaptive EFs for all cycles are plotted in Figure 24. As can be seen, these curves present different shapes due to different speeding profiles, but all the EFs fluctuate around the initial optimal constant $s_{0}$ (1.229) within a small range, which implies the critical role of the stable constant in determining the EF. Meanwhile, the quantitative analysis of Figure 24 indicates that the PI terms account for less than 8.1 percent of EF value, which once again demonstrates the primary function of $s_{0}$ in determining the EF. Moreover, since the integral term in EF takes smaller proportion compared with the stable component, therefore, to develop a strategy in a more efficient way, the adaptive law can be further simplified by eliminating the integral term and just remaining the stable constant and a proportional term, despite slightly sacrificing the solution accuracy. 


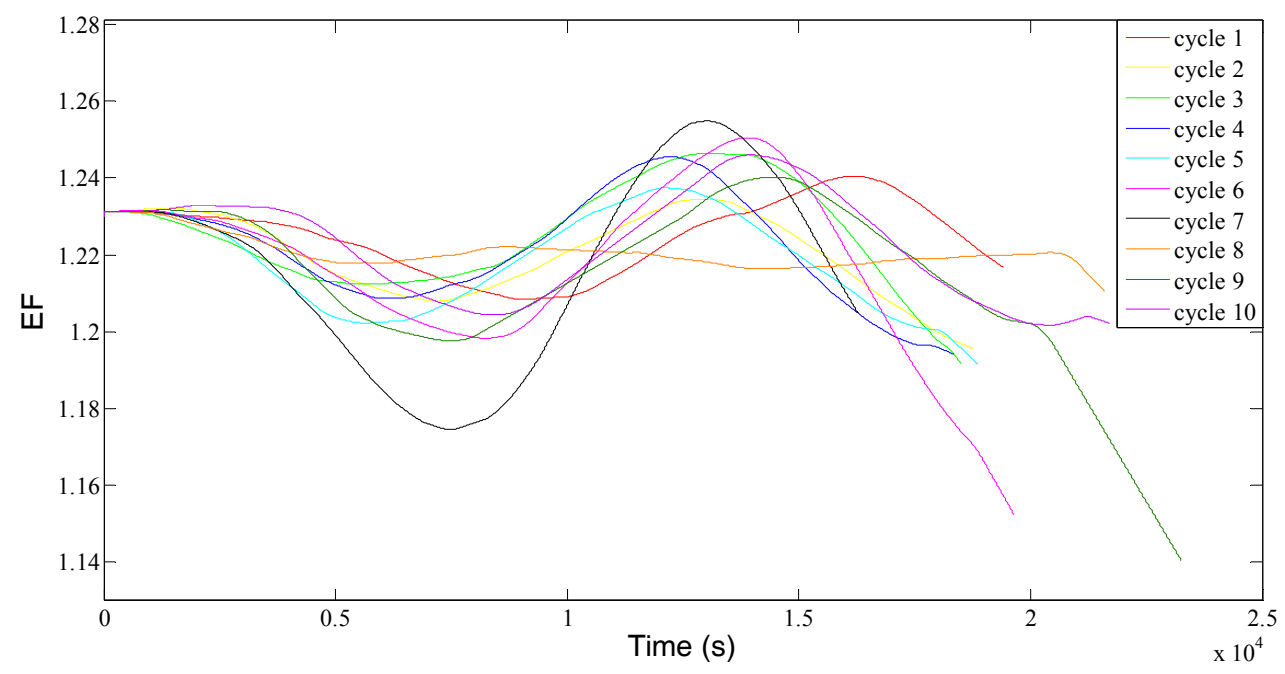

Figure 24. Optimal EFs for the 10 driving cycles.

\section{Conclusions}

To develop a robust A-ECMS, it is of great importance to dynamically tune the EF. Based on the PMP method, this paper proposes a convenient and effective approach of determining the EF for a plug-in hybrid electric bus running on a fixed route and the main conclusions are summarized as follows:

(1) The adaptive EF is considered as a combination of two parts including the major component and calibrating component, in which the major component defined as a constant has a dominate role in determining the EF while the latter denoted as a proportional and integral terms is designed to apply to the disparity of regular speeding profiles.

(2) The numerical solution of PMP strategy is acquired by using the shooting method, and the optimal initial co-state value captured in the last shooting operation is taken as the optimal stable constant, with which it is quite easy to tune the two PI coefficients. Meanwhile, to avoid repeatedly selecting of the initial co-state value in the beginning and obtain the optimal solution as soon as possible, the Secant method is employed to tune the initial co-state variable, which greatly reduces the shooting times and also exhibits a flexibility of adapting any appropriate initial co-state value.

(3) The presented A-ECMS method is examined by ten successive speeding profiles, as well as with three initial SOC levels to simulate different charge levels. The results indicate almost the identical energy consumption cost compared to the DP and PMP algorithms, and the examination with different initial SOCs further demonstrates the robustness of the proposed method. Therefore, the proposed method offers an efficient approach of developing the A-ECMS strategy for the PHEBs with fixed routes.

The future research will establish a more accurate battery model by considering the cooling and heating system and further focus on the engineering application of the strategy.

Acknowledgments: This work was supported by the Fundamental Research Funds for the Central Universities of China (Grand no 310822151026 and 310822151121) and Shaanxi Science and Technology Programming of Innovation Project (Grand no 2015zs-06).

Author Contributions: S.X. conceived and designed the energy management strategy and solved the critical computational issue in the model simulation and wrote the manuscript; H.L. conducted the numerical simulation; Z.X. and T.L. contributed to discussions and analyses of the results; L.W. gave many useful suggestions.

Conflicts of Interest: The authors declare no conflict of interest. 


\section{References}

1. Hu, X.; Martinez, C.M.; Yang, Y. Charging, power management, and battery degradation mitigation in plug-in hybrid electric vehicles: A unified cost-optimal approach. Mech. Syst. Signal Process. 2017, 87, 4-16. [CrossRef]

2. Liu, Y.; Li, J.; Ye, M.; Qin, D.; Zhang, Y.; Lei, Z. Optimal Energy Management Strategy for a Plug-in Hybrid Electric Vehicle Based on Road Grade Information. Energies 2017, 10, 412. [CrossRef]

3. Gonder, J.; Markel, T. Energy Management Strategies for Plug-In Hybrid Electric Vehicles; SAE Permissions: Warrendale, PA, USA, 2007.

4. Hu, X.; Jiang, J.; Egardt, B.; Cao, D. Advanced power-source integration in hybrid electric vehicles: Multicriteria optimization approach. IEEE Trans. Ind. Electron. 2015, 62, 7847-7858. [CrossRef]

5. Serrao, L.; Onori, S.; Rizzoni, G. ECMS as a realization of Pontryagin's minimum principle for HEV control. In Proceedings of the American Control Conference, St. Louis, MO, USA, 10-12 June 2009; pp. 3964-3969.

6. Khayyam, H.; Bab-Hadiashar, A. Adaptive intelligent energy management system of plug-in hybrid electric vehicle. Energy 2014, 69, 319-335. [CrossRef]

7. Schouten, N.J.; Salman, M.A.; Kheir, N.A. Fuzzy logic control for parallel hybrid vehicles. IEEE Trans. Control Syst. Technol. 2002, 10, 460-468. [CrossRef]

8. Lin, C.C.; Peng, H.; Grizzle, J.W.; Kong, J.-M. Power management strategy for a parallel hybrid electric truck. IEEE Trans. Control Syst. Technol. 2003, 11, 839-849.

9. Pisu, P.; Rizzoni, G. A comparative study of supervisory control strategies for hybrid electric vehicles. IEEE Trans. Control Syst. Technol. 2007, 15, 506-518. [CrossRef]

10. Kim, N.; Cha, S.; Peng, H. Optimal control of hybrid electric vehicles based on Pontryagin's minimum principle. IEEE Trans. Control Syst. Technol. 2011, 19, 1279-1287.

11. Xu, L.; Yang, F.; Li, J.; Ouyang, M.; Hua, J. Real time optimal energy management strategy targeting at minimizing daily operation cost for a plug-in fuel cell city bus. Int. J. Hydrogen Energy 2012, 37, 15380-15392. [CrossRef]

12. Hou, C.; Ouyang, M.; Xu, L.; Wang, H. Approximate Pontryagin's minimum principle applied to the energy management of plug-in hybrid electric vehicles. Appl. Energy 2014, 115, 174-189. [CrossRef]

13. Lacandia, F.; Tribioli, L.; Onori, S.; Rizzoni, G. Adaptive energy management strategy calibration in PHEVs based on a sensitivity study. SAE Int. J. Alt. Power 2013, 2, 443-455. [CrossRef]

14. Zhang, F.; Liu, H.; Hu, Y.; Xi, J. A supervisory control algorithm of hybrid electric vehicle based on adaptive equivalent consumption minimization strategy with fuzzy PI. Energies 2016, 9, 919. [CrossRef]

15. Xu, L.; Li, J.; Ouyang, M.; Hua, J.; Yang, G. Multi-mode control strategy for fuel cell electric vehicles regarding fuel economy and durability. Int. J. Hydrogen Energy 2014, 39, 2374-2389. [CrossRef]

16. Tang, L.; Rizzoni, G.; Onori, S. Energy management strategy for HEVs including battery life optimization. IEEE Trans. Transp. Electrification 2015, 1, 211-222. [CrossRef]

17. Tulpule, P.; Marano, V.; Rizzoni, G. Energy management for plug-in hybrid electric vehicles using equivalent consumption minimization strategy. Int. J. Electric Hybrid Veh. 2010, 2, 329-350. [CrossRef]

18. Zhang, F.; Xi, J.; Langari, R. Real-Time energy management strategy based on velocity forecasts using V2V and V2I communications. IEEE Trans. Intell. Transp. Syst. 2017, 18, 416-430. [CrossRef]

19. Sciarretta, A.; Back, M.; Guzzella, L. Optimal control of parallel hybrid electric vehicles. IEEE Trans. Control Syst. Technol. 2004, 12, 352-363. [CrossRef]

20. Onori, S.; Serrao, L. On Adaptive-ECMS strategies for hybrid electric vehicles. In Proceedings of the International Scientific Conference on Hybrid and Electric Vehicles, Malmaison, France, 6-7 December 2011.

21. Gu, B.; Rizzoni, G. An adaptive algorithm for hybrid electric vehicle energy management based on driving pattern recognition. In Proceedings of the International Mechanical Engineering Congress and Exposition, Chicago, IL, USA, 5-10 November 2006; pp. 249-258.

22. Yokoi, Y.; Ichikawa, S.; Doki, S.; Okuma, S.; Naitou, T.; Miki, N. Driving pattern prediction for an energy management system of hybrid electric vehicles in a specific driving course. In Proceedings of the 30th Annual Conference of IEEE Industrial Electronics Society, Busan, Korea, 2-6 November 2004; pp. 1727-1732.

23. Onori, S.; Tribioli, L. Adaptive Pontryagin's Minimum Principle supervisory controller design for the plug-in hybrid GM Chevrolet Volt. Appl. Energy 2015, 147, 224-234. [CrossRef] 
24. Sivertsson, M.; Eriksson, L. Design and evaluation of energy management using map-based ECMS for the PHEV benchmark. Oil Gas Sci. Technol. 2015, 70, 195-211. [CrossRef]

25. Larsson, V.; Johannesson, L.; Egardt, B.; Lasson, A. Benefit of route recognition in energy management of plug-in hybrid electric vehicles. In Proceedings of the American Control Conference, Montreal, QC, Canada, 27-29 June 2012; pp. 1314-1320.

26. Li, L.; Yang, C.; Zhang, Y.; Zhang, L.; Song, J. Correctional DP-based energy management strategy of plug-in hybrid electric bus for city-bus route. IEEE Trans. Veh. Technol. 2015, 64, 2792-2803. [CrossRef]

27. Han, J.; Park, Y.; Kum, D. Optimal adaptation of equivalent factor of equivalent consumption minimization strategy for fuel cell hybrid electric vehicles under active state inequality constraints. J. Power Sources 2014, 267, 491-502. [CrossRef]

28. Borhan, H.; Vahidi, A.; Phillips, A.M. MPC-based energy management of a power-split hybrid electric vehicle. IEEE Trans. Control Syst. Technol. 2012, 20, 593-603. [CrossRef]

29. Hemi, H.; Ghouili, J.; Cheriti, A. Combination of Markov chain and optimal control solved by Pontryagin's Minimum Principle for a fuel cell/supercapacitor vehicle. Energy Convers. Manag. 2015, 91, 387-393. [CrossRef]

30. Xie, S.; He, H.; Peng, J. An energy management strategy based on stochastic model predictive control for plug-in hybrid electric buses. Appl. Energy 2017, 196, 279-288. [CrossRef]

31. Li, G.; Zhang, J.; He, H. Battery SOC constraint comparison for predictive energy management of plug-in hybrid electric bus. Appl. Energy 2017, 194, 578-587. [CrossRef]

32. Li, L.; You, S.; Yang, C.; Yan, B.; Song, J.; Chen, Z. Driving-behavior-aware stochastic model predictive control for plug-in hybrid electric buses. Appl. Energy 2016, 162, 868-879. [CrossRef]

33. Sun, C.; Hu, X.; Moura, S.J.; Sun, F. Comparison of velocity forecasting strategies for predictive control in HEVs. In Proceedings of the ASME 2014 Dynamic Systems and Control Conference, San Antonio, TX, USA, 22-24 October 2014.

34. Sun, C.; Moura, S.J.; Hu, X.; Hedrick, J.K.; Sun, F. Dynamic traffic feedback data enabled energy management in plug-in hybrid electric vehicles. IEEE Trans. Control Syst. Technol. 2015, 23, 1075-1086.

35. Johnson, V.H. Battery performance models in ADVISOR. J. Power Sources 2002, 110, 321-329. [CrossRef]

(C) 2017 by the authors. Licensee MDPI, Basel, Switzerland. This article is an open access article distributed under the terms and conditions of the Creative Commons Attribution (CC BY) license (http:/ / creativecommons.org/licenses/by/4.0/). 